Poweld, E. O. (1958). J. gen. Microbiol. 18, 382-417

\title{
An Outline of the Pattern of Bacterial Generation Times
}

\author{
BY E. O. POWELL
}

Microbiological Research Establishment (Ministry of Supply) Porton, Wiltshire

SUMMARY: The generation times of four species of organisms have been measured, each under several sets of conditions: Aerobacter cloacae, Serratia marcescens, Streptococcus faecalis and Pseudomonas aeruginosa. Minor variations in the experimental conditions appear to affect the mean generation time less in large samples than in small. This can be explained as a result of association between the generation times of closely related organisms. Positive correlation between the generation times of sisters, cousins and perhaps second cousins shows that the influence of an ancestor is felt through two or three generations. The observed correlation between mothers and daughters is usually small, probably because of bias due to the interval between fission of cytoplasm and fission of cell wall. The coefficient of variation of generation time is not a constant for the species but it is stable under given circumstances. It is possibly related systematically to the chemical complexity of the growth medium. In unhampered growth, less than $1 \%$ of the organisms produced are non-viable. There is positive association between the viabilities of sisters, and between the viability of an organism and the generation time of its mother. The distribution of generation times can be represented by a Pearson Type III or else a Pearson Type V distribution; both are convenient in applications.

The generation time of an individual is considered to be determined partly by molecular accidents, partly by heredity.

In a previous paper on this subject (Powell, 1955), I gave an account of some preliminary work designed to test and compare the hypotheses of Rahn (1932) and Kendall $(1948,1952)$ about the causes of variability in the generation times $(\tau)$ of individual bacteria. It turned out that a comparison of the observed distributions of $\tau$ with those predicted by the hypotheses was not altogether satisfactory, and I suggested that a further test might be made by determining the effect of alterations in temperature and growth medium on the coefficient of variation of $\tau$. At the same time I concluded that such experiments should await improvements in observational technique. There appeared to be a possibility that the relevance of the observations was altogether vitiated by 'delayed fission', that is, by the lapse of an appreciable and variable interval between the termination of the processes considered by Rahn and Kendall, and the overt occurrence of fission.

The results which I now present consist of generation time measurements on unicellular organisms of four species-Aerobacter cloacae, Serratia marcescens, Streptococcus faecalis and Pseudomonas aeruginosa-each under several different growth conditions. The undertaking was originally encouraged by the development of an improved culture chamber (Powell, 1956a), which would permit much greater accuracy in the determination of times of fission. At first, I hoped that the results would be of enhanced diacritical value, but it soon became clear that the generation times of bacteria do not depend on chance in the 
simple way implied by the hypotheses of Rahn and Kendall. The $\tau$ in any one population are interrelated in a complex manner, and in the present state of knowledge can fairly be said to be useless for the purpose of establishing the truth of these or any other analogous hypotheses. So far from losing in interest, the results offer new mathematical and biological problems.

My conclusions, though reached by way of a good deal of arithmetic, are almost entirely of a descriptive character. It would be inappropriate at the

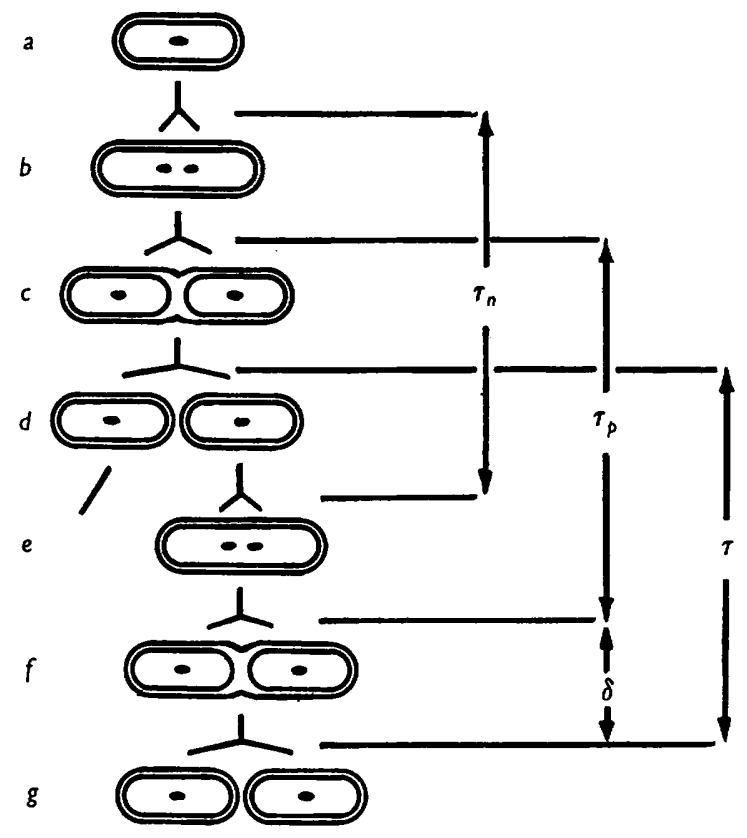

Fig. 1. $a \ldots g$ : stages in fission of a unicellular organism. $\tau_{n}, \tau_{p}, \tau$ : generation times for nucleus, cytoplasm and cell wall respectively. $\delta:$ interval between cytoplasm fission and cell-wall fission.

moment to attempt any quantitative treatment of the behaviour of a given species under given conditions, and I have presented a broad picture as if it were quite generally true. I believe that it is in fact true of many species under a wide range of growth conditions, but $I$ have no doubt that each several inference will be demonstrably falsified in some instances.

'I have so far used the expression 'generation time' in its ordinary sensea vague one-and before proceeding to a description of the experiments and the analysis of data, I wish to give it a more precise meaning. The fission of a unicellular organism can be imagined to take place in three obvious stages; these are represented in Fig. 1. At $(a)$ a single organism is shown as bounded by the cell wall (outer oval); within it the cell membrane (inner oval) encloses the cytoplasm, and the nucleus appears as a central dot. It is evident that when fission occurs, first the nucleus $(b)$, then the cytoplasm $(c)$, then the cell wall $(d)$, must divide. Only at $(d)$ will two mechanically independent particles be produced, though it is likely that the two protoplasts at $(c)$ are 
organically autonomous. These stages are repeated in the first generation progeny $(e, f, g$; only one daughter cell is shown in Fig. 1 ), and they give rise to three 'generation times': $\tau_{n}, \tau_{p}, \tau$, the intervals between successive fissions of nuclei, cytoplasm, and cell wall, respectively. The morphology and behaviour of the bacterial nucleus is still a matter of controversy. Fission of the nucleus has never been observed; it is not known to be of the unitary character that Fig. 1 suggests, nor is it known that the genetic copying mechanism is carried solely by the nucleus. Accordingly I exclude $\tau_{n}$ from further consideration. The interval $\tau$ is that which is measured in the present experiments, i.e. the experimentally determined times of fission correspond to separation into mechanically independent pieces. Where necessary, I distinguish the events terminating intervals $\tau_{p}$ and $\tau$ as ' $p$-fission' and ' $o$-fission' respectively; otherwise, 'fission', 'inception', 'termination' and 'generation time' are to be understood to refer to, or be determined by, $o$-fission. (Most cells are invested with at least a thin mucous capsule, and a pair of sisters may remain attached thereby after the completion of fission. But the capsule has probably no vital function, and such a pair can be separated without damage by means of a micromanipulator. The capsule does not interfere with the observation of fission in the foregoing sense.) The interval $\delta$ (Fig. 1) between a $p$-fission and the succeeding $o$-fission is accessible, though with difficulty, to observation, and in certain cases it can be shown that $p$-fission is considerably the earlier (Powell, 1956a), though no quantitative work has yet been done. It is likely that $\delta$ is always of appreciable magnitude and variance, and I think therefore that Rahn's expression 'delayed fission' as applied to normal cells should not be perpetuated, since it suggests abnormality.

Under the action of certain drugs (e.g. $m$-cresol, acridine dyestuffs, excess of magnesium, radioactivity) most Gram-negative organisms will grow into long filaments which have little or no obvious structure unless they are stained (Pl. 1, a). When the filaments are treated with a hypertonic saline solution, the cytoplasm shrinks and can then be seen to be divided into a number of short pieces (Pl. 1, $b$ ); apparently $o$-fission has been inhibited, but $p$-fission proceeds at nearly the normal rate. Unless the saline treatment is prolonged, the filaments will recover their apparent continuity and continue to grow when the original medium is replaced. When growth is resumed in the absence of the inhibitor, the filaments will at the same time fairly quickly break down into units of normal length, and some at least of the points of fission correspond to gaps which have appeared under the action of saline. Sometimes a portion of a filament will collapse, lose its optical contrast, and grow no further. This is another indication of the existence of independent protoplasts within the elongated cell wall. It seems therefore that $o$-fission is a phenomenon of secondary importance in growth; certainly it ought not to be too readily assumed to be linked directly with fundamental rate-controlling processes. We can expect only a rough correspondence between the two generation times $\tau_{p}$ and $\tau$. 


\title{
DESCRIPTION OF THE EXPERIMENTS
}

\author{
Method
}

The organisms were grown under the microscope in an improved culture chamber (Powell, 1956a), which permitted observation at a magnification of $\times 1500$ with transmitted phase-contrast illumination. The medium was constantly circulated through the chamber. The experiments were nearly all conducted in the same way: the chamber was inoculated from an overnight culture of the organism to be examined, and left for a period equal to 5 or 6 mean generation times, enough to establish steady growth and a stable agedistribution. (This period, if not known roughly from experience, was estimated in a pilot experiment.) By this time small colonies had been formed; the cover-glass of the chamber was lifted and replaced so as to break them up. Then a field containing a few well-separated single organisms was selected and left for a further 15-30 min. before observation was begun.

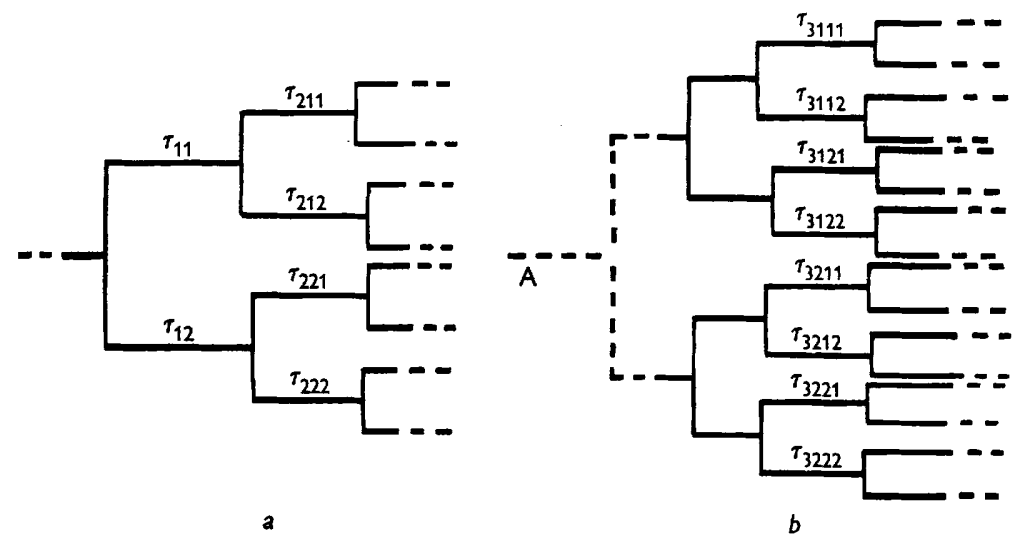

Fig. 2. Typical family trees. The complete horizontal segments represent measured generation times.

The growth of the selected organisms was followed for two complete generations so that each yielded a set of six generation times (two daughters and four granddaughters); Fig. $2 a$ shows the typical family tree. The field was watched continuously at critical periods so that the actual times of fission (that is, of separation into apparently discrete pieces) could be estimated; previously the observations had been made at fixed and regular intervals. The generation times, i.e. the intervals between successive fissions, were recorded to the nearest $2 \mathrm{~min}$. or $5 \mathrm{~min}$, according as the average $\tau$ was below or above $50 \mathrm{~min}$. The $\tau$ values quoted are the means for these groups. With the new optical system, I am confident that few generation times have been assigned to the wrong group as a result of errors of judgement.

During the waiting period after the disturbance of the cover-glass, it frequently happened that some of the selected cells divided. In such cases both daughter cells were followed for two further generations. Each pair thus 
provided twelve generation times, in two sets of six known to be related as in Fig. $2 b$, though the termination of the original ancestor $A$ was not observed.

For conciseness, it is convenient to have a special terminology for sets of organisms (or their generation times) which are related in a particular way. I adopt the following: a ' $H$-triad' is a mother and two daughters such as $\tau_{11}$, $\tau_{211}, \tau_{212}$ in Fig. $2 a$; $A$ ' $F$-hexad' is a family of six related as are $\tau_{11}, \tau_{12}, \tau_{211}$, $\tau_{212}, \tau_{221}, \tau_{222}$; ' ' $S$-dyad' is a pair of sisters such as $\tau_{11}, \tau_{12}$; a ' $C_{1}$-tetrad' is a set of first cousins such as $\tau_{2 a b}(a, b=1,2)$; and a ' $C_{2}$-octad' is a set of second cousins such as $\tau_{3 a b c}(a, b, c=1,2)$ in Fig. $2 b$.

In a few experiments, especially where the generation times were very long, observation was continued for one generation only; and in all there were some incomplete sets resulting from the occurrence of non-viable organisms and the escape of motile organisms from the field of view. Each series of observations carried out on a given species under given cultural conditions was continued until a sample of 300-450 individual generation times had been accumulated.

Organisms which have been under observation with phase-contrast illumination in the new chamber can at any time be stained in situ without appreciable disturbance. This feature was taken advantage of in some ancillary experiments to determine the morphological status of fission as seen under phasecontrast illumination. Organisms at different stages of development were treated with specific cell-wall stains (see, for example, Bisset, 1955; Tomcsik \& Grace 1955); it was found that the time of separation into discrete pieces corresponded approximately to the cell-wall fission-that is, to $o$-fission, stage $(d)$ of Fig. 1.

\section{Organisms and media}

The following is a list of the growth media used in the experiments:

Media TMB and PW. Conventional tryptic meat broth and peptone water.

Medium $H$. Devised by $\mathrm{Dr} D$. Herbert (private communication). The medium is made up from three solutions which are stored separately at $0^{\circ}$ in Pyrex bottles.

Solution (i): $\mathrm{NaH}_{2} \mathrm{PO}_{4} \cdot 2 \mathrm{H}_{2} \mathrm{O}, 31 \mathrm{~g}$; $\left(\mathrm{NH}_{4}\right)_{2} \mathrm{HPO}_{4}, 238 \mathrm{~g}$.; $\mathrm{K}_{2} \mathrm{SO}_{4}, 70$ g.; water to 11 .

Solution (ii): $\mathrm{MgO}, 4 \cdot 00$ g. ; $\mathrm{CaCO}_{3}, 2 \cdot 00$ g. $; \mathrm{FeSO}_{4} .7 \mathrm{H}_{2} \mathrm{O}, 0 \cdot 56$ g.; $\mathrm{ZnSO}_{4} .7 \mathrm{H}_{2} \mathrm{O}$, $0 \cdot 29$ g.; $\mathrm{MnSO}_{4} .4 \mathrm{H}_{2} \mathrm{O}, 0.22 \mathrm{~g}$.; $\mathrm{CuSO}_{4} .5 \mathrm{H}_{2} \mathrm{O}, 0.05 \mathrm{~g}$.; concentrated $\mathrm{HCl}$ solution $25 \mathrm{ml}$; ; water to 11 .

Solution (iii): Glucose, $20 \mathrm{~g}$., water to $100 \mathrm{ml}$.

'Analar' chemicals and demineralized water were used for making up the solutions: batches of the complete medium were freshly prepared as required by mixing: solution (i) $50 \mathrm{ml}$; ; solution (ii) $5 \mathrm{ml}$.; solution (iii) $50 \mathrm{ml}$.; water $895 \mathrm{ml}$.

Medium HP. Medium $\mathrm{H}$ with the addition of $0.1 \%(\mathrm{w} / \mathrm{v})$ recrystallized sodium pyruvate, introduced as the solid immediately before use.

Medium HG. Medium $\mathrm{H}$ with the addition of $0 \cdot 15 \%(\mathrm{w} / \mathrm{v})$ L-glutamic acid. Medium HT. Medium $\mathrm{H}$ with the addition of $0.05 \%(\mathrm{w} / \mathrm{v})$ sodium thiosulphate pentahydrate. 
Medium HGST. Medium $\mathrm{H}$ with the addition of $0.1 \%(\mathrm{w} / \mathrm{v})$ succinic acid, $0.15 \%(\mathrm{w} / \mathrm{v})$ L-glutamic acid, $0.05 \%(\mathrm{w} / \mathrm{v})$ sodium thiosulphate pentahydrate.

It was found necessary to pay great attention to general cleanliness and to the purity and freshness of the chemically defined media, if steady and reproducible growth was to be achieved.

It will be seen that medium $\mathbf{H}$ contains glucose, ammonia and sulphate as the sole sources of carbon, nitrogen and sulphur, respectively; the various additions made offer these elements in alternative and for some purposes more readily assimilable forms.

The sixteen series of experiments are set out in Table 1, where they are each given a symbol for convenient reference in later tables. The column headed 'oxygen supply' refers to the gas used to drive the medium-circulating pump,

Table 1. Organisms and grozth conditions

\begin{tabular}{|c|c|c|c|c|c|}
\hline $\begin{array}{l}\text { Series } \\
\text { reference } \\
\text { symbol }\end{array}$ & Organism & Medium & $\begin{array}{c}\text { Tem- } \\
\text { perature } \\
\left({ }^{\circ} \mathrm{C} .\right)\end{array}$ & $\begin{array}{c}\text { Oxygen } \\
\text { supply }\end{array}$ & $\begin{array}{l}\text { No. of } \\
\text { experi- } \\
\text { ments }\end{array}$ \\
\hline $\left.\begin{array}{r}\mathrm{Ba} \\
1 \\
2 \\
3 \\
4 \\
5 \\
6\end{array}\right\}$ & Aerobacter cloacae & $\left\{\begin{array}{l}\text { TMB } \\
\text { TMB } \\
\text { H } \\
\text { HP } \\
\text { HG } \\
\text { HT }\end{array}\right.$ & $\begin{array}{l}30 \\
35 \\
35 \\
35 \\
35 \\
35\end{array}$ & $\begin{array}{l}\text { Air } \\
\text { Air, } O_{2} \\
\text { Air, } O_{2} \\
\text { Air, } O_{2} \\
\text { Air } \\
\text { Air }\end{array}$ & $\begin{array}{r}10 \\
9 \\
12 \\
9 \\
9 \\
9\end{array}$ \\
\hline $\left.\begin{array}{r}\text { Cp } 1 \\
2 \\
3 \\
4 \\
5\end{array}\right\}$ & Serratia marcescens & $\left\{\begin{array}{l}\text { TMB } \\
\mathrm{H} \\
\mathrm{HP} \\
\mathrm{HG} \\
\mathrm{H}^{\prime} \mathrm{T}\end{array}\right.$ & $\begin{array}{l}\mathbf{3 5} \\
\mathbf{3 5} \\
\mathbf{3 5} \\
\mathbf{3 5} \\
\mathbf{3 5}\end{array}$ & $\begin{array}{l}\text { Air } \\
\text { Air } \\
\text { Air } \\
\text { Air } \\
\text { Air }\end{array}$ & $\begin{array}{l}\mathbf{9} \\
\mathbf{9} \\
\mathbf{9} \\
\mathbf{9} \\
\mathbf{8}\end{array}$ \\
\hline $\left.\begin{array}{r}\text { Sf } 1 \\
2 \\
3\end{array}\right\}$ & Streptococcus faecalis & $\left\{\begin{array}{l}\text { TMB } \\
\text { TMB } \\
\text { TMB }\end{array}\right.$ & $\begin{array}{l}30 \\
35 \\
\mathbf{3 5}\end{array}$ & $\begin{array}{l}\text { Air } \\
\text { Air } \\
\mathrm{O}_{2}\end{array}$ & $\begin{array}{r}9 \\
11 \\
9\end{array}$ \\
\hline $\left.\begin{array}{rr}P p \\
2\end{array}\right\}$ & Pseudomonas aeruginosa & $\left\{\begin{array}{l}\text { PW } \\
\text { HSGT }\end{array}\right.$ & $\begin{array}{l}35 \\
35\end{array}$ & $\begin{array}{l}\text { Air } \\
\text { Air }\end{array}$ & $\begin{array}{l}11 \\
10\end{array}$ \\
\hline
\end{tabular}

ultimately the source of the oxygen required by the organisms for respiration and growth. Series $\mathrm{Ba} 2,3$ and 4 each comprise two sets of experiments. In one set the pump was driven by pure oxygen, so that the concentration of dissolved gas was increased. A statistical test showed that neither the generation time nor its variance was significantly altered thereby; thus there was no evidence of inadequate oxygenation, and the two sets were combined. Series Sf 3 is treated separately because the higher oxygen concentration increased both the generation time and its variance very significantly (Powell, 1956a). The inocula in series $\mathrm{Ba} \mathrm{1,2}$ and 3; $\mathrm{Cp} \mathrm{1;} \mathrm{Sf} \mathrm{1,} 2$ and 3; $\mathrm{Pp} 1$ and 2, were drawn from cultures maintained on the same media as were used in the experiments themselves; the numerical results for these series probably represent the stable behaviour of the species concerned. This is not necessarily true of the other series. The strain of Serratia marcescens chosen was fully chromogenic when grown on complex media, but began to throw off colourless variants

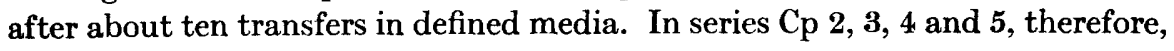


a mother culture was maintained in tryptic meat broth; inocula from it were subcultured thrice only in medium $\mathrm{H}$ before use. In series $\mathrm{Ba} 4,5$ and 6 the culture was maintained in medium $\mathbf{H}$; it is to be noted, then, that in these three series and in $\mathrm{Cp} \mathrm{3,4}$ and 5 the organisms under observation were in contact with the fortified media HP, HG and HT for the the first time. Pseudomonas aeruginosa grew slowly and uncertainly on the synthetic media with or without a single addendum; medium HSGT, with three, supported rapid growth. There appears to be no easily prepared defined medium suitable for Streptococcus faecalis.

\section{The uniformity of Cellophane}

The general conclusions of this paper depend on a single ostensible fact, that organisms closely related by descent tend to have similar generation times. Now since such organisms lie in close juxtaposition on the Cellophane (Messrs British Cellophane Ltd.) membrane of the culture chamber, the similarity could conceivably be a mere casual result of inequalities in the permeability of the membrane; organisms overlying a relatively impermeable patch would be poorly nourished. I had therefore originally planned an extensive series of tests for uniformity. However, during the course of the investigations different experiments, both of my own and of independent workers, showed that other properties of bacteria were interrelated in a similar way to the generation times. I think now that the following considerations are sufficient rebuttal of any doubts on this score:

(i) Under the phase-contrast microscope, Cellophane is very uniform in its apparent optical density, except that it contains a great number of welldefined ellipsoidal inclusions, nearly all less than $5 \mu$. across. These are too small, too frequent and too uniformly disposed to be a cause of impermeability on the required scale (they are not of course necessarily impermeable themselves). Fields showing large inclusions near to the surface are naturally avoided in experimental work ( $\mathrm{Pl} .1, c)$.

(ii) The polarizing microscope also shows no evidence of phase changes such as might be expected in areas of high density.

(iii) If organisms lying on Cellophane are stained by means of dyes applied to the distal surface of the membrane, there is no obvious inequality in the depth of colour attained. Yet the dye molecules are much larger than any in the defined culture media.

(iv) From a generation time distribution it is possible to calculate the growth rate to be expected in a whole culture in the same medium and at the same temperature. In the few cases where comparison with a directly and accurately determined growth rate has been made, the two values have agreed within about $2 \%$, and that determined from the generation time distribution has been the greater (but not significantly so). The average permeability of Cellophane is thus high enough not to restrict the access of nutrilites to the inoculum; if there are patches of effectively low permeability, their total area must be small.

(v) Most enzyme reactions, and in fact the reactions of intact organisms 
with their nutrilites, obey a law of the Michaelis type. If $S$ is the concentration of a substrate or nutrilite, $t$ the time, and $k$ and $K$ are constants,

$$
-\frac{d S}{d t}=k \frac{S}{K+S}
$$

Usually $K$ is exceedingly small, and $d S / d t$ is nearly constant for $S \gg K$ (compare, for example, Dagley \& Hinshelwood, 1938), so moderate inequalities in the permeability of the membrane should not affect the growth rate, unless the passage of nutrilites is everywhere much impeded.

\section{Constancy of growth rate}

I have previously pointed out that the distribution of generation times can be usefully investigated only when the growth rate is constant. In each series of experiments I therefore carried out a rather crude test for constancy of growth rate: most experiments were continued for a time sufficient to include two successive generations of organisms; evidently the mean for the first generation (the mothers) should be the same as the mean for the second generation (the daughters). Taking all the experiments of one series together, suppose there are $n_{m}$ mothers, of mean $\bar{\tau}_{m}$, and $n_{d}$ daughters, of mean $\bar{\tau}_{d}$. Under the null hypothesis that both generations have the same distribution of $\tau$, we can take for $\operatorname{var} \tau$ the value obtained from the whole sample, and carry out a $t$-test for the significance of $\left|\bar{\tau}_{m}-\bar{\tau}_{d}\right|$. Normally we should have

$$
\operatorname{var}\left(\bar{\tau}_{m}-\bar{\tau}_{d}\right)=\left(1 / n_{m}+1 / n_{d}\right) \operatorname{var} \tau \text {, }
$$

but here the real variance of the difference is much greater because of correlation between generation times of related organisms. The principal factor which increases the variance is the strong positive correlation between sisters (Table 4B). Now almost without exception both mothers and daughters occur as $S$-dyads in the sample (cf. Fig. 2), and if $\tau_{11}, \tau_{12}$, are the generation times of a dyad,

$$
\begin{aligned}
\operatorname{var}\left(\tau_{11}+\tau_{12}\right) & =2\left\{\operatorname{var} \tau+\operatorname{cov}\left(\tau_{11}, \tau_{12}\right)\right\} \\
& =2 \operatorname{var} \tau\{1+\rho(S)\},
\end{aligned}
$$

where $\rho(S)$ is the coefficient of correlation between sisters. Thus the variance of the mean for the pair is greater than $(\operatorname{var} \tau) / 2$ by the factor $1+\rho(S)$. The variance of the mean for a sample of $n$, consisting of $S$-dyads only, and in the absence of other appreciable correlations, is not $(\operatorname{var} \tau) / n$ but $\{1+\rho(S)\}(\operatorname{var} \tau) / n$. Hence we have more nearly

$$
\operatorname{var}\left(\bar{\tau}_{m}-\bar{\tau}_{d}\right)=\left(1 / n_{m}+1 / n_{d}\right)\{1+\rho(S)\} \operatorname{var} \tau .
$$

Example 1. Series $B a 1$

$$
\begin{aligned}
\bar{\tau}_{m}=30 \cdot 39 ; \quad n_{m}=123 . \\
\bar{\tau}_{d}=29 \cdot 41 ; \quad n_{d}=246 . \\
\left.\bar{\tau}_{m}-\bar{\tau}_{d}=0.98 ; \quad \text { var } \tau=36 \cdot 92 \text { (Table } 10\right) . \\
\text { Estimate of } \rho(S)=0.58 \text { (Table } 4) . \\
\left(1 / n_{m}+1 / n_{d}\right) \text { var } \tau=0.4502, \\
\text { var } \left.\bar{\tau}_{m}-\bar{\tau}_{d}\right)=0.7098, \\
\text { Student's } t=1 \cdot 18 ; P(t)=0.24 .
\end{aligned}
$$


In four of the sixteen series thus tested, the $t$-probability was below the $5 \%$ level, but of these four, three exhibited appreciable negative mother-daughter correlation (Series Ba 2, Cp 3, Pp 1, Table 4C). Such correlation tends to exaggerate the difference between the means. As the sign of $\bar{\tau}_{m}-\bar{\tau}_{d}$ was positive in eight series, negative in the other eight, and as a correction for the very appreciable correlation between cousins (Table $4 \mathrm{D}$ ) would have reduced the $t$-values still further, I have assumed throughout that the growth rates have been constant.

\section{ANALYSIS OF THE DATA}

The example of the previous section shows how very much the failure of independence within the sample can exaggerate the result of a significance test. The effect is tantamount to a decrease in the number of degrees of freedom. In the two sections next following I have applied significance tests in the conventional way without any allowance for internal correlation, and it will be seen that the probability levels reached are consequently often widely scattered, and include many very small values (internal correlation affects the apparent probability level most when the true value is already rather small). These tests are therefore useful for comparison only; the general inferences are based on uniformity of behaviour. The type of argument adopted may be expressed formally thus: on a null hypothesis $H$, the possible outcomes of a trial are $P$ and $N$, say, each with probability $\frac{1}{2} ; n$ trials are made, and $p_{0}$ result in $P$. Then if $p_{0}$ differs sufficiently from $\frac{1}{2} n, \sim H$ is asserted. The probability of $p \leqslant p_{0}$ or $p \geqslant p_{0}$ for a given $n$ can be read from tables of partial sums of the binomial series (e.g. National Bureau of Standards, 1949).

The treatment which $I$ have been able to devise for the purposes of these sections is solely directed to the demonstration of results of the above kind. It is very desirable that some less clumsy kind of manipulation should be developed. The product-moment coefficient of correlation, in particular, seems inappropriate; but it has the virtues of familiarity and arithmetical simplicity.

\section{Stability of sample means; heterogeneity}

In my previous investigation, I submitted the data for each series to a twoway analysis of variance for 'within experiment' and 'between experiment' components, and found that the latter was significantly in excess, presumably because of lack of reproducibility of the growth-rate (or mean generation time). This appears to be true here also; see Example 2.

The variance $\sigma_{E}^{2}$ thus estimated I call the 'apparent inter-experiment variance'; it is a measure of the amount by which the mean generation time differs from one experiment to another. The sixteen values are listed in Table 2, A, but the $F$-ratios and their probabilities are not given because the latter are all below 0.001. The obvious conclusion is unduly pessimistic, however. To continue with the same data as in Example 2, a test for homogeneity was carried out by dividing the nine experiments into two groups, such that the number of observations in any experiment of the 'upper' group was greater than the number in any experiment of the 'lower' group. Two-way 
Example 2. Series $B a 2$

2-way analysis of variance

No. of experiments $=k=9$.

No. of observations in $j$ th expt. $=n_{j}=21,24,31,31,36,47,48,64,70$.

Total no. of observations $=N=372$.

$\begin{array}{ccccc}\begin{array}{c}\text { Source of } \\ \text { variance }\end{array} & \begin{array}{c}\text { Sum of } \\ \text { squares }\end{array} & \begin{array}{c}\text { Degrees } \\ \text { of freedom }\end{array} & \begin{array}{c}\text { Mean } \\ \text { square }\end{array} & \begin{array}{c}\text { Variance } \\ \text { estimated }\end{array} \\ \text { Between experiments } & 1,169 & 8 & 146 \cdot 1 & \sigma_{\tau}^{2}+M \sigma_{B}^{2} \\ \text { Within experiments } & 8,968 & 363 & 24.71 & \sigma_{\tau}^{2} \\ \text { Total } & 10,137 & 371 & - & - \\ M=\frac{N^{2}-\Sigma n_{j}^{2}}{N(k-1)}=40 \cdot 538 ; F \text {-ratio }=5.91(8,363) ; P(F)<0 \cdot 001 \text {; estimated } \sigma_{E}^{2}=2 \cdot 99 .\end{array}$

Table 2. Tro-way analyses of variance

A : estimated inter-experiment variance, all experiments together. B: $\boldsymbol{F}$-ratio (and degrees of freedom), $F$-ratio probability, and estimated inter-experiment variance for largesample experiments. $C$ : the same for small-sample experiments. In $A$, the $\sigma_{E}^{2}$ are all significant at the 0.001 level. (See Examples 2 and 3.)

\begin{tabular}{|c|c|c|c|c|c|c|c|}
\hline \multirow[b]{2}{*}{ Series } & \multicolumn{2}{|l|}{$\mathbf{A}$} & \multicolumn{2}{|l|}{$\mathbf{B}$} & \multicolumn{3}{|c|}{$\mathrm{C}$} \\
\hline & Est. $\sigma_{I}^{2}$ & $F\left(n_{1}, n_{2}\right)$ & $P(F)$ & Est. $\sigma_{E U}^{2}$ & $F\left(n_{1}, n_{2}\right)$ & $P(F)$ & Est. $\sigma_{E L}^{2}$ \\
\hline $\mathrm{Ba} 1$ & $6 \cdot 27$ & $5.95(4,209)$ & $<0.001$ & $4 \cdot 18$ & $13 \cdot 65(4,153)$ & $<0.001$ & $10 \cdot 5$ \\
\hline 2 & 2.99 & $3 \cdot 32(3,225)$ & $<0.025$ & $1 \cdot 08$ & $8 \cdot 82(4,138)$ & $<0 \cdot 001$ & $6 \cdot 07$ \\
\hline 3 & $11 \cdot 4$ & $5.92(4,206)$ & $<0.001$ & $7 \cdot 56$ & $5 \cdot 21(6,100)$ & $<0.001$ & $21 \cdot 6$ \\
\hline 4 & $11 \cdot 9$ & $6.52(3,246)$ & $<0.001$ & $9 \cdot 79$ & $4 \cdot 84(4,139)$ & $<0.001$ & $20 \cdot 5$ \\
\hline 5 & $2 \cdot 10$ & $3 \cdot 08(3,198)$ & $<0.001$ & $0 \cdot 926$ & $8 \cdot 03(4,116)$ & $<0.001$ & $4 \cdot 96$ \\
\hline 6 & $8 \cdot 71$ & $11 \cdot 03(3,223)$ & $<0.001$ & $7 \cdot 07$ & $17 \cdot 02(4,146)$ & $<0.001$ & $11 \cdot 7$ \\
\hline P 1 & $9 \cdot 48$ & $34 \cdot 48(3,205)$ & $<0.001$ & $6 \cdot 89$ & $21 \cdot 33(4,153)$ & $<0.001$ & $14 \cdot 0$ \\
\hline 2 & $31 \cdot 3$ & $5.90(3,241)$ & $<0.001$ & $15 \cdot 1$ & $8.59(4,127)$ & $<0.001$ & $73 \cdot 5$ \\
\hline 3 & $88 \cdot 2$ & $1 \cdot 23(3,225)$ & $>0.25$ & $1 \cdot 06$ & $20 \cdot 04(4,142)$ & $<0.001$ & 167 \\
\hline 4 & $27 \cdot 1$ & $17 \cdot 57(3,249)$ & $<0.001$ & $27 \cdot 2$ & $17 \cdot 14(4,140)$ & $<0.001$ & $36 \cdot 0$ \\
\hline $\mathbf{5}$ & $14 \cdot 9$ & $4 \cdot 84(3,224)$ & $<0.005$ & $9 \cdot 24$ & $5 \cdot 35(3,887)$ & $<0.005$ & $33 \cdot 2$ \\
\hline ff 1 & $6 \cdot 44$ & $8 \cdot 39(3,202)$ & $<0.001$ & $1 \cdot 69$ & $25 \cdot 39(4,153)$ & $<0.001$ & $12 \cdot 4$ \\
\hline 2 & $4 \cdot 63$ & $29 \cdot 09(4,274)$ & $<0.001$ & $4 \cdot 81$ & $25 \cdot 76(5,157)$ & $<0.001$ & $5 \cdot 52$ \\
\hline 3 & $5 \cdot 23$ & $27 \cdot 27(3,268)$ & $<0.001$ & $3 \cdot 88$ & $12 \cdot 22(4,169)$ & $<0.001$ & $8 \cdot 67$ \\
\hline 1 & $6 \cdot 28$ & $5 \cdot 28(4,250)$ & $<0.001$ & $2 \cdot 09$ & $18 \cdot 79(5,104)$ & $<0.001$ & $16 \cdot 6$ \\
\hline 2 & $4 \cdot 81$ & $5 \cdot 24(3,200)$ & $<0.005$ & $2 \cdot 50$ & $2 \cdot 18(5,119)$ & $>0.05$ & 0.95 \\
\hline
\end{tabular}

analysis of both groups separately gave the suggestive result that the interexperiment variance was greater in the lower group $\left(\sigma_{E L}^{2}\right)$ than in the upper $\left(\sigma_{E U}^{2}\right)$ (see Example 3).

When this test was repeated with the other series, it was found that in only one case was $\sigma_{E L}^{2}$ less than $\sigma_{E U}^{2}$ (Table $2, \mathrm{~B}, \mathrm{C}$ ). If on the average $\sigma_{E L}^{2}$ were equal to $\sigma_{E U}^{2}$, the probability of so great a disparity would be $0 \cdot 0005$. It seems therefore that the mean of small samples is less stable than that of large, and that the apparent fluctuation is in part caused by some form of heterogeneity in the population. It may be inferred that if still larger samples could be handled in single experiments, the $\sigma_{E}^{2}$ would be still smaller. While it is too much to hope that the fluctuation resulting from lack of reproducibility is really zero, the inter-experiment variance is probably less than the $\sigma_{E U}^{2}$ of 


\section{Example 3. Series $B a 2$}

2-roay analysis of upper group

\begin{tabular}{|c|c|c|c|c|}
\hline \multicolumn{5}{|c|}{$k=4 ; n_{j}=47,48,64,70 ; N=229$} \\
\hline $\begin{array}{c}\text { Source of } \\
\text { variance }\end{array}$ & $\begin{array}{l}\text { Sum of } \\
\text { squares }\end{array}$ & $\begin{array}{l}\text { Degrees } \\
\text { of freedom }\end{array}$ & $\begin{array}{c}\text { Mean } \\
\text { square }\end{array}$ & $\begin{array}{l}\text { Variance } \\
\text { estimated }\end{array}$ \\
\hline Between experiments & 263 & $\mathbf{3}$ & $87 \cdot 67$ & $\sigma_{\tau U}^{2}+M \sigma_{Z U}^{2}$ \\
\hline Within experiments & 5933 & 225 & $26 \cdot 37$ & $\sigma_{\tau U}^{2}$ \\
\hline Total & 6196 & 228 & - & - \\
\hline \multicolumn{5}{|c|}{$M=56.67 ; F$-ratio $=3.32(3,225) ; P(F)<0.001 ;$ estimated $\sigma_{E U}^{2}=1.08$} \\
\hline \multicolumn{5}{|c|}{ 2-way analysis of lower group } \\
\hline \multicolumn{5}{|c|}{$k=5 ; n_{j}=21,24,31,31,36 ; N=143$} \\
\hline $\begin{array}{l}\text { Source of } \\
\text { variance }\end{array}$ & $\begin{array}{l}\text { Sum of } \\
\text { squares }\end{array}$ & $\begin{array}{c}\text { Degrees } \\
\text { of freedom }\end{array}$ & $\begin{array}{c}\text { Mean } \\
\text { square }\end{array}$ & $\begin{array}{l}\text { Variance } \\
\text { estimated }\end{array}$ \\
\hline Between experiments & 776 & 4 & $194 \cdot 00$ & $\sigma_{\tau L}^{2}+M \sigma_{E L}^{2}$ \\
\hline Within experiments & 3035 & 138 & $21 \cdot 99$ & $\sigma_{\tau L}^{2}$ \\
\hline Total & 3811 & 142 & - & - \\
\hline
\end{tabular}

Table 2, B, and in most cases is probably a small fraction of the population variance (compare the $\sigma_{E U}^{2}$ of Table 2, B with the estimated $\mu_{2}$ of Table 10). In what follows, therefore, I assume that the true inter-experiment variance lies between $\sigma_{E}^{2}$ and 0 . So far this assumption is only an extrapolation. However, the dependence of apparent inter-experiment variance on sample size can be plausibly explained as a result of positive association between the generation times of nearly related organisms (and more specific reasons for adopting this explanation will appear in the next section). The selection of organisms for observation is not a random process; reference to the section on method will show that it must frequently happen that all the organisms selected at the beginning of an experiment come from one clone, the progeny of a single cell of the inoculum; and this is more likely to be true, the smaller the number of organisms chosen-a large number is more likely to derive from several clones. The explanation is in accord with the impression given by the earlier work, viz. 'a very large family tree, representing the numerical and temporal extension of a culture, consists of a patchwork of areas within some of which growth is steady, while within others it is erratic' (Powell, 1955); it can be tested by an extension of the analysis of variance. If we call a group of closely related organisms a 'family', we can determine by a three-way analysis the significant existence of inter-family as well as inter-experiment variances. It is obviously convenient to choose as the family units the $F$-hexads (Fig. $2 a$ ) into which most of the observations were naturally grouped (see Example 4).

In eleven of the sixteen series, the $\boldsymbol{F}_{F}$-ratio probability was below 0.05 , and in only two was the ratio itself less than unity - fairly strong evidence of a real inter-family variance (Table $\mathbf{3}, \mathbf{A}$ ). In this analysis we have, by admitting a variance $\sigma_{E 3}^{2}$, proceeded on the assumption that appreciable inter-experiment 


\section{Example 4. Series $C p 4$ \\ 3-way analysis of variance}

No. of experiments $=k=9$.

No. of $F$-hexads in $j$ th expt. $=n_{j}=12,12,8,5,5,4,4,4,2$.

Total no. of observations $=N=6 \Sigma n_{j}=336$.

\begin{tabular}{|c|c|c|c|c|}
\hline $\begin{array}{l}\text { Source of } \\
\text { variance }\end{array}$ & $\begin{array}{l}\text { Sum of } \\
\text { squares }\end{array}$ & $\begin{array}{l}\text { Degrees } \\
\text { of freedom }\end{array}$ & $\begin{array}{l}\text { Mean } \\
\text { square }\end{array}$ & $\begin{array}{l}\text { Variance } \\
\text { estimated }\end{array}$ \\
\hline Between experiments & 7,749 & 8 & $968 \cdot 6$ & $\sigma_{\tau 8}^{2}+6 \sigma_{73}^{2}+M \sigma_{\mathbb{Z} 3}^{2}$ \\
\hline $\begin{array}{l}\text { Within experiments } \\
\text { and between hexads }\end{array}$ & 9,226 & 47 & $196 \cdot 3$ & $\sigma_{\tau 8}+6 \sigma_{F 3}^{2}$ \\
\hline Within hexads & 17,850 & 280 & $63 \cdot 75$ & $\sigma_{\tau 3}^{2}$ \\
\hline Total & $\mathbf{3 4 ; 8 2 5}$ & 335 & - & - \\
\hline \multicolumn{5}{|c|}{$M=6 \frac{\left(\Sigma n_{j}\right)^{2}-\Sigma n_{j}^{2}}{\left(\Sigma n_{j}\right)(k-1)}=35.920$} \\
\hline \multicolumn{5}{|c|}{ 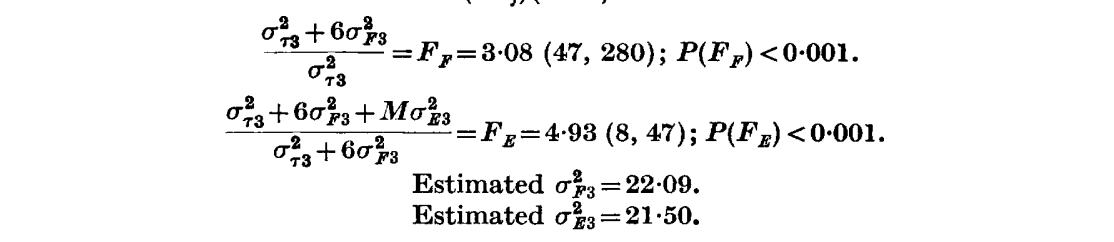 } \\
\hline
\end{tabular}

variance exists. But there is no reason to suppose that the $F$-hexad has any special significance, and if, as is likely, the association extends beyond the limits of this group, part of the $\sigma_{E 3}^{2}$ will be contributed by inter-family variance. Assuming for the time being that the real inter-experiment variance is zero, we can combine the first two lines of the 3-way analysis of Example 4.

\section{Example 5. Series $C p 4$ \\ 2-way analysis of variance}

No. of $\boldsymbol{F}$-hexads $=56$.

No. of observations $=336$.

\begin{tabular}{|c|c|c|c|c|}
\hline $\begin{array}{l}\text { Source of } \\
\text { variance }\end{array}$ & $\begin{array}{l}\text { Sum of } \\
\text { squares }\end{array}$ & $\begin{array}{c}\text { Degrees of } \\
\text { freedom }\end{array}$ & $\begin{array}{c}\text { Mean } \\
\text { square }\end{array}$ & $\begin{array}{l}\text { Variance } \\
\text { estimated }\end{array}$ \\
\hline Between hexads & 16,975 & 55 & $308 \cdot 63$ & $\sigma_{\tau 2}^{2}+6 \sigma_{F 2}^{2}$ \\
\hline Within hexads & 17,850 & 280 & $63 \cdot 75$ & $\sigma_{\tau 2}^{2}$ \\
\hline Total & 34,825 & 335 & - & 一 \\
\hline
\end{tabular}

The estimates of $\sigma_{F 2}^{2}$ so found are given in Table 3, B. We may tentatively regard $\sigma_{F^{2}}^{2}$ and $\sigma_{F 3}^{2}$ as upper and lower limits for a quantity, the 'inter-family variance', which measures in a gross but comprehensive way the extent to which generation times are associated as a result of hereditary effects. (I do not imply that the inheritance is necessarily nuclear or chromosomal in character.)

Inspection of the records suggests also that properly conducted tests for homogeneity of variance would disclose similar regularities in the relation between sample size and the Bartlett criterion. Unfortunately the significance of the criterion can be very much altered by a correction for the kurtosis of the 


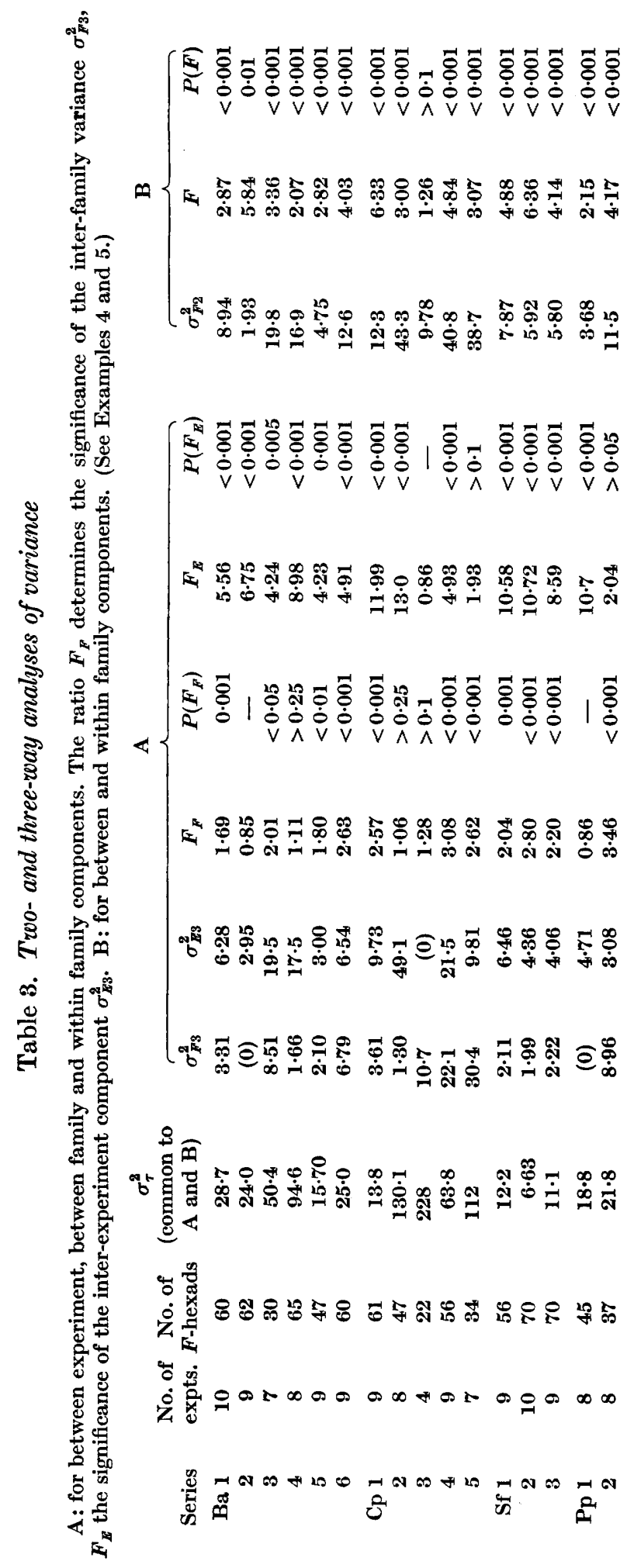


presumptive population (see, for example, Box, 1953). Here the kurtosis is so poorly determined as to be quite unreliable; the sample values of $\gamma_{2}$ (Table 10) range from $-0 \cdot 2$ to $8 \cdot 1$, and show no obvious association with species or experimental conditions. For the purpose of estimating fourth moments the sample sizes are already small, and are effectively reduced further by internal correlation. Hence in the absence of any acceptable hypothesis about the population distribution, no conclusions can be drawn from the Bartlett test.

\section{Correlation between generation times}

Four correlations having a simple significance can be computed from the data available, viz. the correlations between mothers and daughters $(\rho(\boldsymbol{H}))$, between sisters $(\rho(S))$, between first cousins $\left(\rho\left(C_{1}\right)\right)$ and between second cousins $\left(\rho\left(C_{2}\right)\right)$. But before the results can be presented, certain difficulties require discussion.

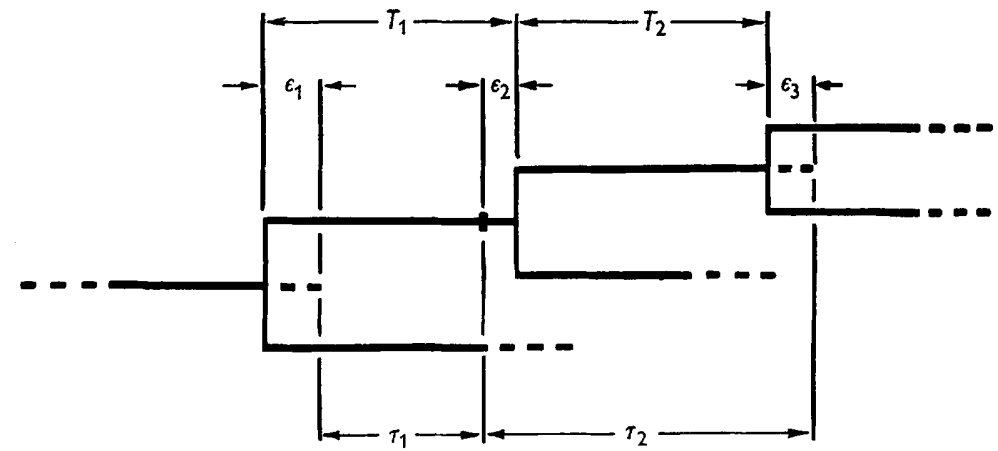

Fig. 3. Effect of errors, $\epsilon$, on correlation between generation times. $\tau_{1}, \tau_{2}$ : measured values; $T_{1} T_{2}$; true values.

In Fig. 3, $T_{1}$ and $T_{2}$ represent the true generation times of a mother and a daughter cell respectively. If the corresponding estimated times of fission are in error by $\epsilon_{1}, \epsilon_{2}, \epsilon_{3}$, the recorded generation times will be $\tau_{1}, \tau_{2}$, where

$$
\begin{aligned}
& \tau_{1}=T_{1}+\epsilon_{2}-\epsilon_{1}, \\
& \tau_{2}=T_{2}+\epsilon_{3}-\epsilon_{2} .
\end{aligned}
$$

Now if we assume as we reasonably may, that the $\epsilon$ are all distributed alike and are independent of the $\boldsymbol{T}$ and of one another,

$$
\begin{aligned}
\operatorname{var} \tau & =\operatorname{var} T+2 \operatorname{var} \epsilon, \\
\operatorname{var}\left(\tau_{1}+\tau_{2}\right) & =2 \operatorname{var} T+2 \operatorname{cov}\left(T_{1}, T_{2}\right)+2 \operatorname{var} \epsilon,
\end{aligned}
$$

and the observed mother-daughter correlation will be

$$
\begin{aligned}
\rho(H) & =\frac{\operatorname{var}\left(\tau_{1}+\tau_{2}\right)}{2 \operatorname{var} \tau}-1 \\
& =\frac{\operatorname{cov}\left(T_{1}, T_{2}\right)-\operatorname{var} \epsilon}{\operatorname{var} T+2 \operatorname{var} \epsilon} .
\end{aligned}
$$


Thus $\rho(H)$ will be biased towards the value $-\frac{1}{2}$ by the common error $\left(\epsilon_{2}\right)$ which affects $\tau_{1}$ and $\tau_{2}$ in opposite ways. Analogous reasoning shows that the correlation between sisters is biased towards $+\frac{1}{2}$ by the common error in determining the time of inception:

$$
\rho(S)(\text { observed })=\frac{\operatorname{cov}\left(T_{1}, T_{2}\right)+\operatorname{var} \epsilon}{\operatorname{var} T+2 \operatorname{var} \epsilon},
$$

where now $T_{1}, T_{2}$ refer to the true generation times of an $S$-dyad. Because of the uncertain magnitude of $\operatorname{var} \epsilon$, I have computed estimates of $\rho(\boldsymbol{H})$ and $\rho(S)$ from the crude moments of the paired observations, but it is highly improbable that 2 var $\epsilon$ much exceeds the usual Sheppard correction for grouping (which it includes) or that its neglect had led to wrong conclusions. However, this point will need closer attention when it becomes possible to treat $\rho(\boldsymbol{H})$ and $\rho(S)$ in quantitative detail.

The correlation coefficients are also subject to a bias (always towards positive values) as a result of inter-experiment variance. We may consider (cf. Powell, $1955)$ the generation times measured in any one experiment to be a sample from a distribution $h\left(\tau, a, c_{0}\right)$ whose mean $a$ varies from experiment to experiment but whose coefficient of variation $c_{0}$ is constant. (In the next section it will be seen that there are good grounds for this assumption.) Let $j(a)$ be the frequency function of the mean, $c_{j}$ its coefficient of variation, and let $a$ and $c$ be the grand mean and coefficient of variation of all the observations. Then

$$
c^{2}+1=\left(c_{0}^{2}+1\right)\left(c_{j}^{2}+1\right),
$$

and

$$
a=\iint \tau h\left(\tau, a, c_{0}\right) j(a) d a d \tau=\int a j(a) d a .
$$

Now in a given experiment let

$$
\mathrm{H}\left(\tau_{1}, \tau_{2} ; a, a ; c_{0}, c_{0} ; \rho_{0}\right)
$$

be the joint distribution of pairs of generation times $\tau_{1}, \tau_{2}$ (of a particular sort), and assume for the time being that the correlation $\rho_{0}$ is constant, or at least statistically independent of $a$. Write $\mu, \mu(h), \mu(\mathrm{H})$ for the moments of the overall distribution and of the $h$ - and $\mathrm{H}$-distributions. Then

$$
\left.\begin{array}{l}
\mu_{10}^{\prime}(\mathrm{H})=\mu_{01}^{\prime}(\mathrm{H})=\mu_{1}^{\prime}(h)=a, \\
\mu_{20}^{\prime}(\mathrm{H})=\mu_{02}^{\prime}(\mathrm{H})=\mu_{2}^{\prime}(h)=a^{2}\left(c_{0}^{2}+1\right), \\
\mu_{11}(\mathrm{H})=\rho_{0} \mu_{2}(h), \\
\mu_{11}^{\prime}(\mathrm{H})=a^{2}\left(\rho_{0} c_{0}^{2}+1\right) .
\end{array}\right\}
$$

The value of the correlation coefficient calculated from all the observations will be an estimate of

$$
\frac{\mu_{11}}{\mu_{2}}=\rho, \text { say. }
$$

But

$$
\begin{aligned}
\mu_{11}^{\prime} & =\int a^{2}\left(\rho_{0} c_{0}^{2}+1\right) j(a) d a \quad(\text { from }(5)) \\
& =a^{2}\left(\rho_{0} c_{0}^{2}+1\right)\left(c_{j}^{2}+1\right) ; \\
\mu_{11} & =a^{2}\left(\rho_{0} c_{0}^{2} c_{j}^{2}+\rho_{0} c_{0}^{2}+c_{j}^{2}\right),
\end{aligned}
$$

while

$\mu_{2}=a^{2} c^{2}$. 
Hence, using (4) or

$$
\begin{aligned}
\rho & =\rho_{0}+\left(1-\rho_{0}\right) c_{j}^{2} / c^{2}, \\
\rho_{0} & =\rho-(1-\rho) c_{j}^{2} /\left(c^{2}-c_{j}^{2}\right) .
\end{aligned}
$$

(It may be noted that $c_{j}^{2} / c^{2}=\operatorname{var} a / \operatorname{var} \tau$, where var $a$ is the inter-experiment variance.)

There is no very good reason to suppose that $\rho_{0}$ really is independent of $a$, though the constancy of $c_{0}$ suggests it. However, we may safely deny a null hypothesis that $\rho_{0} \equiv 0$ if $\rho$ differs sufficiently from $c_{j}^{2} / c^{2}$. If, on the other hand, apparently $\rho=c_{j}^{2} / c^{2}$ it does not follow that $\rho_{0} \equiv 0$, since the observed equality could be produced by a $\rho_{0}$ varying appropriately with $a$.

There is some difficulty in the choice of a coefficient to represent the correlation between cousins and between second cousins. Two measures suggest themselves immediately: (i) we may compute correlations $\rho\left(C_{1}\right), \rho\left(C_{2}\right)$, from all possible pairs; a $C_{1}$-tetrad provides four pairs, a $C_{2}$-octad sixteen (cf. Fig. 2), but not all are independent; (ii) we may compute correlations $\rho\left(\bar{C}_{1}\right), \rho\left(\bar{C}_{2}\right)$ between the means of the members on either side of the family, so that each tetrad or octad yields only one pair. The advantage of $\rho\left(C_{1}\right), \rho\left(C_{2}\right)$ is that the correction for inter-experiment variance (Equations 5 and 6) applies to them directly. Moreover, pairs from incomplete tetrads and octads may be included in the sample. But, like the usual intraclass correlation coefficient, they are restricted in range; in fact

$$
\begin{aligned}
& \left|\rho\left(C_{1}\right)\right| \leqslant \frac{1}{2}\{1+\rho(S)\} ; \\
& \left|\rho\left(C_{2}\right)\right| \leqslant \frac{1}{4}\{1+\rho(S)\}\left\{1+\rho\left(\bar{C}_{1}\right)\right\} .
\end{aligned}
$$

The correlations $\rho\left(\bar{C}_{1}\right), \rho\left(\bar{C}_{2}\right)$ between means have the full possible range -1 to +1 . Corresponding to Equation (6) we have for the effect of inter-experiment variance

$$
\begin{aligned}
& \rho\left(\bar{C}_{1}\right)=\rho_{0}\left(\bar{C}_{1}\right)+\left\{1-\rho_{0}\left(\bar{C}_{1}\right)\right\} \frac{2 c_{j}^{2}}{c^{2}\{1+\rho(S)\}}, \\
& \rho\left(\bar{C}_{2}\right)=\rho_{0}\left(\bar{C}_{2}\right)+\left\{1-\rho_{0}\left(\bar{C}_{2}\right)\right\} \frac{4 c_{j}^{2}}{c^{2}\{1+\rho(S)\}\left\{1+\rho\left(\bar{C}_{1}\right)\right\}} .
\end{aligned}
$$

So far as the whole population is concerned, these formulae are a mere algebraic transformation of Equation (6), (with $\rho=\rho\left(C_{1}\right)$ etc.), but when the $\rho$ are replaced by sample values, they become very sensitive to sampling fluctuation because of the extra factors in the last terms on the right. Consequently $I$ have preferred the coefficients $\rho\left(C_{1}\right), \rho\left(C_{2}\right)$, formed from all possible pairs of cousins and second cousins. These coefficients, because of their limited range, reflect only weakly the similarity between the two branches of a family stemming from one ancestor.

In order not to give a wrong impression of the sample sizes, I have adopted a special notation in Table $4, \mathrm{C}, \mathrm{D}, \mathrm{E}$. Thus a mother-daughter correlation is mostly derived from $H$-triads $\left(\tau_{11}, \tau_{211}, \tau_{212}\right.$; Fig. $\left.2 a\right)$, each yielding two pairs with a common member, but in any series there are some isolated pairs; the sample size is reported as $A_{3}+B_{1}$, where $A$ is the number of $H$-triads and $B$ the number of isolated pairs; the total number of pairs is $2 A+B$. For first cousins, $A_{4}+B_{1}$ means that the sample consists of $A$ complete $C_{1}$-tetrads plus a number 
of variously incomplete tetrads providing $B$ extra pairs; total $4 A+B$. Similarly, for second cousins.

The distribution of the $\tau$ is always markedly skew, and it might at first seem better to compute correlation coefficients from $\log \tau$, which is a nearly normal variate. But for the present merely synoptic purpose the untransformed variate has the advantage that the correlations computed from it are affected in a simple way by the different sources of error.

Table 4. Estimates of the product-moment correlation coefficients between mothers and daughters $(\rho(H))$, sisters $(\rho(S))$, first cousins $\left(\rho\left(C_{1}\right)\right)$ and second cousins $\left(\rho\left(C_{2}\right)\right)$

The factor $c_{j}^{2} / c^{2}$ is calculated from the $\sigma_{E}^{2}$ of Table $2 \mathrm{~A}$ and the $\mu_{2}$ of Table 10.

\begin{tabular}{|c|c|c|c|c|c|c|c|c|c|}
\hline \multirow[b]{2}{*}{ Series } & \multirow{2}{*}{$\begin{array}{c}\mathbf{A} \\
c_{j}^{2} / c^{2}\end{array}$} & \multicolumn{2}{|c|}{ B } & \multicolumn{2}{|r|}{$\mathrm{C}$} & \multicolumn{2}{|r|}{ D } & \multicolumn{2}{|c|}{$\mathbf{E}$} \\
\hline & & $\begin{array}{c}\rho(S) \\
+\end{array}$ & $\begin{array}{c}\text { Sample } \\
\text { size }\end{array}$ & $\rho(\boldsymbol{H})$ & $\begin{array}{l}\text { Sample } \\
\text { size }\end{array}$ & $\begin{array}{c}\rho\left(C_{2}\right) \\
+\end{array}$ & $\begin{array}{l}\text { Sample } \\
\text { size }\end{array}$ & $\rho\left(C_{2}\right)$ & $\begin{array}{c}\text { Sample } \\
\text { size }\end{array}$ \\
\hline $\begin{array}{r}\text { Ba } 1 \\
2 \\
\mathbf{2} \\
4 \\
4 \\
5 \\
6\end{array}$ & $\begin{array}{l}0 \cdot 170 \\
0 \cdot 111 \\
0 \cdot 151 \\
0 \cdot 0896 \\
0 \cdot 0970 \\
0 \cdot 222\end{array}$ & $\begin{array}{l}0 \cdot 576 \\
0 \cdot 603 \\
0 \cdot 355 \\
0 \cdot 606 \\
0 \cdot 609 \\
0 \cdot 419\end{array}$ & $\begin{array}{l}185 \\
184 \\
155 \\
195 \\
161 \\
186\end{array}$ & $\begin{array}{l}-0.036 \\
-0.316 \\
+0.166 \\
-0.093 \\
+0.015 \\
+0.312\end{array}$ & $\begin{array}{r}122_{3}+2_{1} \\
127_{3}+1_{1} \\
65_{3}+5_{1} \\
131_{3}+1_{1} \\
97_{3} \\
125_{3}+6_{1}\end{array}$ & $\begin{array}{l}0 \cdot 411 \\
0 \cdot 207 \\
0 \cdot 256 \\
0 \cdot 306 \\
0 \cdot 364 \\
0 \cdot 292\end{array}$ & $\begin{array}{c}73_{4}+4_{1} \\
71_{4}+2_{1} \\
47_{4}+5_{1} \\
89_{4}+1_{1} \\
72_{4} \\
85_{4}+9_{1}\end{array}$ & $\begin{array}{l}+0.309 \\
+0 \cdot 099 \\
+0 \cdot 087 \\
+0 \cdot 357 \\
+0 \cdot 164 \\
+0 \cdot 120\end{array}$ & $\begin{array}{c}16_{8}+6_{1} \\
16_{8} \\
13_{8}+20_{1} \\
30_{8}+4_{1} \\
36_{8}+8_{1} \\
23_{8}+50_{1}\end{array}$ \\
\hline $\begin{array}{r}\text { Cp } 1 \\
\mathbf{2} \\
\mathbf{3} \\
4 \\
5\end{array}$ & $\begin{array}{l}0 \cdot 406 \\
0 \cdot 147 \\
0 \cdot 267 \\
0 \cdot 246 \\
0 \cdot 0942\end{array}$ & $\begin{array}{l}0.698 \\
0.570 \\
0.735 \\
0.791 \\
0.592\end{array}$ & $\begin{array}{l}182 \\
187 \\
185 \\
196 \\
159\end{array}$ & $\begin{array}{r}+0.302 \\
+0.014 \\
-0.186 \\
+0.200 \\
+0.085\end{array}$ & $\begin{array}{r}125_{3}+1_{1} \\
96_{3}+3_{1} \\
48_{3}+2_{1} \\
116_{3}+5_{1} \\
69_{3}+1_{1}\end{array}$ & $\begin{array}{l}0.556 \\
0.436 \\
0.496 \\
0 \cdot 680 \\
0.478\end{array}$ & $\begin{array}{l}7 \mathbf{1}_{4}+4_{1} \\
77_{4}+3_{1} \\
80_{4}+7_{1} \\
79_{4}+8_{1} \\
75_{4}+2_{1}\end{array}$ & $\begin{array}{l}+0.199 \\
-0.122 \\
+0.318 \\
+0.391 \\
+0.224\end{array}$ & $\begin{array}{c}13_{8}+8_{1} \\
17_{8}+16_{1} \\
15_{8}+32_{1} \\
23_{8}+36_{1} \\
19_{8}\end{array}$ \\
\hline $\begin{array}{r}\text { Sf } 1 \\
2 \\
3\end{array}$ & $\begin{array}{l}0 \cdot 343 \\
0 \cdot 386 \\
0 \cdot 255\end{array}$ & $\begin{array}{l}0.769 \\
0 \cdot 804 \\
0.758\end{array}$ & $\begin{array}{l}180 \\
220 \\
222\end{array}$ & $\begin{array}{l}+0.152 \\
+0.182 \\
+0.146\end{array}$ & $\begin{array}{c}116_{3}+3_{1} \\
142_{3} \\
142_{3}+1_{1}\end{array}$ & $\begin{array}{l}0 \cdot 610 \\
0 \cdot 629 \\
0 \cdot 493\end{array}$ & $\begin{array}{r}80_{4}+8_{1} \\
106_{4}+2_{1} \\
98_{4}+4_{1}\end{array}$ & $\begin{array}{r}+0.508 \\
+0.335 \\
+0.292\end{array}$ & $\begin{array}{l}21_{8}+21_{1} \\
35_{8}+8_{1} \\
28_{8}+12_{1}\end{array}$ \\
\hline $\begin{array}{r}p 1 \\
2\end{array}$ & $\begin{array}{l}0 \cdot 230 \\
0 \cdot 167\end{array}$ & $\begin{array}{l}0 \cdot 582 \\
0.780\end{array}$ & $\begin{array}{l}181 \\
161\end{array}$ & $\begin{array}{l}-0.120 \\
+0.016\end{array}$ & $\begin{array}{l}95_{3}+3_{1} \\
78_{3}+5_{1}\end{array}$ & $\begin{array}{l}0 \cdot 266 \\
0.418\end{array}$ & $\begin{array}{l}74_{4}+41_{1} \\
71_{4}+10_{1}\end{array}$ & $\begin{array}{l}+0.283 \\
+0.359\end{array}$ & $\begin{array}{l}23_{8}+12_{1} \\
23_{8}+57_{1}\end{array}$ \\
\hline
\end{tabular}

The crude estimates of the four correlations are given in Table 4, B, C, D, E, together with (A) the factor $c_{j}^{2} / c^{2}=\operatorname{var} a / \operatorname{var} \tau$. Here var $a$ is taken as the estimated $\sigma_{E}^{2}$ of Table $2, \mathrm{~A}$, which is almost certainly considerably greater than the true inter-experiment variance, and $\operatorname{var} \tau$ is the estimated $\mu_{2}$ of the whole distribution (Table 10). All the $\rho(S)$ exceed $c_{j}^{2} / c^{2}$, often by a large margin; of the $\rho(H)$ all but two are less than $c_{j}^{2} / c^{2}$, but the differences are on the whole smaller. All the $\rho\left(C_{1}\right)$ also exceed $c_{j}^{2} / c^{2}$ by a moderate amount. The corrected estimates of $\rho_{0}(S), \rho_{0}(\boldsymbol{H})$ in Table $5, \mathbf{A}$ were obtained by application of Equation (7). We see that the correlation between sisters is certainly strongly positive and between cousins it is appreciably so. Between mothers and daughters it is more variable but usually near zero-the uncertainty about the true value of $\sigma_{E}^{2}$ (and so of $c_{j}^{2} / c^{2}$ ) makes it impossible to say whether usually positive or negative. The combination of positive $\rho(S)$ and $\rho\left(C_{1}\right)$, perhaps offset by a small negative $\rho(H)$, itself suggests that the group of six generation times forming a $\boldsymbol{F}$-hexad might exhibit a smaller variance than a group of six chosen at random. The conclusion of the previous section, that an inter-family 
variance exists (or, equally, that the $\tau$ of closely related organisms are associated), is corroborated.

Of the estimates of $\rho\left(C_{2}\right)$ (Table 4, E) all but one are positive, but only ten exceed $c_{j}^{2} / c^{2}$, not in itself an unlikely event. Considering that the adopted value of $c_{j}^{2} / c^{2}$ is probably too large we may perhaps admit a weak implication that second cousins are positively associated.

Table 5. Estimates of correlations corrected for inter-experiment variance $\left(\rho_{0}(S), \rho_{0}(H), \rho_{0}\left(C_{1}\right)\right)$ and for inter-family variance $\left(\rho_{F}(S), \rho_{F}(H)\right)$

The factor $c_{k}^{2} / c^{2}$ is calculated from the $\sigma_{k^{2}}^{2}$ of Table 3,B and the $\mu_{2}$ of Table 10.

\begin{tabular}{|c|c|c|c|c|c|c|}
\hline \multirow[b]{2}{*}{ Series } & \multicolumn{3}{|c|}{$\mathbf{A}$} & B & \multicolumn{2}{|c|}{ C } \\
\hline & $\begin{array}{c}\rho_{0}(S) \\
+\end{array}$ & $\rho_{0}(H)$ & $\begin{array}{c}\rho_{0}\left(C_{1}\right) \\
+\end{array}$ & $c_{k}^{2} / c^{2}$ & $\begin{array}{c}\rho_{F}(S) \\
\quad+\end{array}$ & $\begin{array}{c}\rho_{\boldsymbol{F}}(\boldsymbol{H}) \\
-\end{array}$ \\
\hline Ba 1 & $0 \cdot 490$ & $-0 \cdot 248$ & $0 \cdot 291$ & $0 \cdot 242$ & $0 \cdot 441$ & $0 \cdot 367$ \\
\hline 2 & $0 \cdot 553$ & -0.481 & $0 \cdot 108$ & 0.072 & 0.572 & $0 \cdot 418$ \\
\hline 3 & $0 \cdot 240$ & +0.018 & $0 \cdot 124$ & $0 \cdot 263$ & $0 \cdot 125$ & $0 \cdot 132$ \\
\hline 4 & 0.567 & -0.201 & $0 \cdot 238$ & $0 \cdot 127$ & $0 \cdot 549$ & $0 \cdot 252$ \\
\hline 5 & 0.567 & $-0 \cdot 091$ & $0 \cdot 296$ & 0.219 & 0.500 & $0 \cdot 261$ \\
\hline 6 & $0 \cdot 253$ & $+0 \cdot 115$ & 0.090 & 0.322 & $0 \cdot 144$ & 0.014 \\
\hline Cp 1 & 0.491 & $-0 \cdot 173$ & $0 \cdot 252$ & 0.526 & $0 \cdot 362$ & $0 \cdot 472$ \\
\hline 2 & 0.496 & $-0 \cdot 156$ & $0 \cdot 339$ & $0 \cdot 203$ & $0 \cdot 460$ & $0 \cdot 237$ \\
\hline 3 & 0.638 & -0.618 & $0 \cdot 313$ & 0.030 & 0.727 & $0 \cdot 222$ \\
\hline 4 & 0.722 & -0.061 & 0.576 & 0.370 & $0 \cdot 668$ & $0 \cdot 270$ \\
\hline 5 & 0.550 & -0.010 & 0.424 & 0.245 & $0 \cdot 460$ & $0 \cdot 212$ \\
\hline Sf 1 & $0 \cdot 648$ & $-0 \cdot 290$ & $0 \cdot 406$ & 0.419 & $0 \cdot 603$ & 0.459 \\
\hline 2 & $0 \cdot 680$ & -0.333 & $0 \cdot 395$ & 0.494 & $0 \cdot 612$ & 0.617 \\
\hline 3 & 0.676 & -0.147 & 0.319 & 0.283 & $0 \cdot 663$ & $0 \cdot 192$ \\
\hline Pp 1 & 0.456 & $-0 \cdot 383$ & $0 \cdot 047$ & 0.135 & 0.516 & $0 \cdot 257$ \\
\hline 2 & 0.736 & -0.182 & $0 \cdot 301$ & 0.400 & $0 \cdot 633$ & 0.639 \\
\hline
\end{tabular}

Thus a given organism has a part in determining the particular generation times of its progeny, certainly down to the second, and perhaps to the third generation.

We may now think of the mean $\tau$ of a $F$-hexad as an approximate measure of the local growth rate, and the $\sigma_{F 2}^{2}, \sigma_{F 3}^{2}$ of Table 3 as measures of its fluctuation; it becomes of interest to ask further, what is the strength of the local association (i.e. relative to the local $\bar{\tau}$ ) between sisters and between mothers and daughters? The required estimates may be obtained by applying Equation (7) again to $\rho(S)$ and $\rho(H)$, but now the coefficient of variation of the experiment means, $c_{j}^{2}$, must be replaced by the coefficient of variation of $F$-hexad means, say $c_{k}^{2}$, obtained from the $\sigma_{F 2}^{2}$ of Table $3, \mathrm{~B}$. The two correlations $\rho_{F}(S), \rho_{F}(H)$ so corrected for inter-family variance are given in Table $5, \mathrm{C}$; the $\rho_{F}(H)$ are uniformly negative and generally comparable in magnitude with, though less than, the $\rho_{F}(S)$. This is most unusual. If an organism possesses a regulating mechanism which influences a property of its immediate progeny their similarity to itself is not necessarily secured thereby, but similarity is very generally observed, and this is as true of manners or money as of genes. (Egregious exceptions such as are found in sporozoa and cryptogamia are 
explicable in genetic terms.) It may be objected that the notion of a local growth rate and its attendant variances is factitious, but there is prima facie evidence for its propriety. Comparison of $\rho(H)$ (Table 4, C) with $c_{k}^{2} / c^{2}$ (Table 5, B) suggests that the (algebraically) smaller $\rho(H)$ are associated with the smaller $c_{k}^{2} / c^{2}$; in fact the Spearman rank correlation between them is about $+\mathbf{0 \cdot 8 6}$, significantly different from zero at the 0.001 level. Thus the less the $\rho(\boldsymbol{H})$ are affected by the inter-family variance (of the sample if not of the population) the smaller they appear to be. It seems that the difference in sign of the local correlations $\rho_{F}(S)$ and $\rho_{F}(H)$ is genuine, though their sample values are very poorly determined.

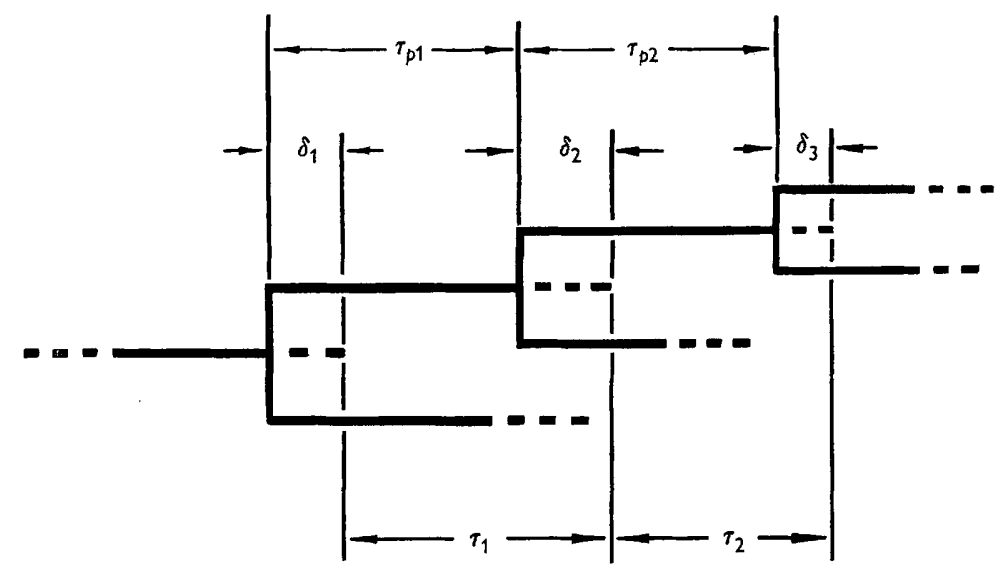

Fig. 4. Relation between observed $o$-generation times $\tau_{1}, \tau_{2}$ and $p$-generation times $\tau_{p 1}, \tau_{p 2}$ as affected by 'delays' $\delta$.

An explanation can be found by an examination of the process of fission (Fig. 1). It rests on the relation between the family tree whose nodes are $p$-fissions (say the $p$-tree) and the tree whose nodes are (observed) $o$-fissions (say the $o$-tree). In Fig. 4, the solid line represents part of a $p$-tree; the intervals between fissions, $\tau_{p}$, have the same significance as in Fig. 1. The $o$-fissions are separated by intervals $\delta$ from the corresponding $p$-fissions. (I assume for the time being that every $\delta$ is less than the $\tau_{p}$ with the same origin.) The $\tau$, intervals between successive $o$-fissions, are the observed generation times. Then if $\tau_{1}, \tau_{2}$ refer to mother and daughter,

$$
\begin{aligned}
\operatorname{var}\left(\tau_{1}+\tau_{2}\right)= & \operatorname{var} \tau_{p 1}+\operatorname{var} \tau_{p 2}+\operatorname{var} \delta_{1}+\operatorname{var} \delta_{3}+2 \operatorname{cov}\left(\tau_{p 1}, \tau_{p 2}\right)+2 \operatorname{cov}\left(\tau_{p 1}, \delta_{3}\right) \\
& +2 \operatorname{cov}\left(\tau_{p 2}, \delta_{3}\right)-2 \operatorname{cov}\left(\tau_{p 1}, \delta_{1}\right)-2 \operatorname{cov}\left(\tau_{p 2}, \delta_{1}\right)-2 \operatorname{cov}\left(\delta_{1}, \delta_{3}\right), \quad(8) \\
\operatorname{var} \tau= & \operatorname{var} \tau_{p}+2 \operatorname{var} \delta+2 \operatorname{cov}\left(\tau_{p 1}, \delta_{2}\right)-2 \operatorname{cov}\left(\tau_{p 1}, \delta_{1}\right)-2 \operatorname{cov}\left(\delta_{1}, \delta_{2}\right) .
\end{aligned}
$$

If now the covariances between the $\delta$, and between them and the $\tau_{p}$ are neglected, and the variances are referred to the local mean $\tau$,

$$
\rho_{F}(H)=\frac{\operatorname{cov}\left(\tau_{p 1}, \tau_{p 2}\right)-\operatorname{var} \delta}{\operatorname{var} \tau_{p}+2 \operatorname{var} \delta}
$$


and similarly (precisely as in Equations (2) and (3)) for sisters

$$
\rho_{F}(S)=\frac{\operatorname{cov}\left(\tau_{p 1}, \tau_{p 2}\right)+\operatorname{var} \delta}{\operatorname{var} \tau_{p}+2 \operatorname{var} \delta}
$$

Because of the symmetry of the joint distribution, $\operatorname{cov}\left(\tau_{p 1}, \tau_{p 2}\right) \leqslant \operatorname{var} \tau_{p}$ and we have only to allow that $\operatorname{var} \tau_{p}<\operatorname{var} \delta$ to make $\rho_{F}(H)$ negative, even though we might expect the covariances in Equations (9) and (10) to be both positive and of similar magnitude.
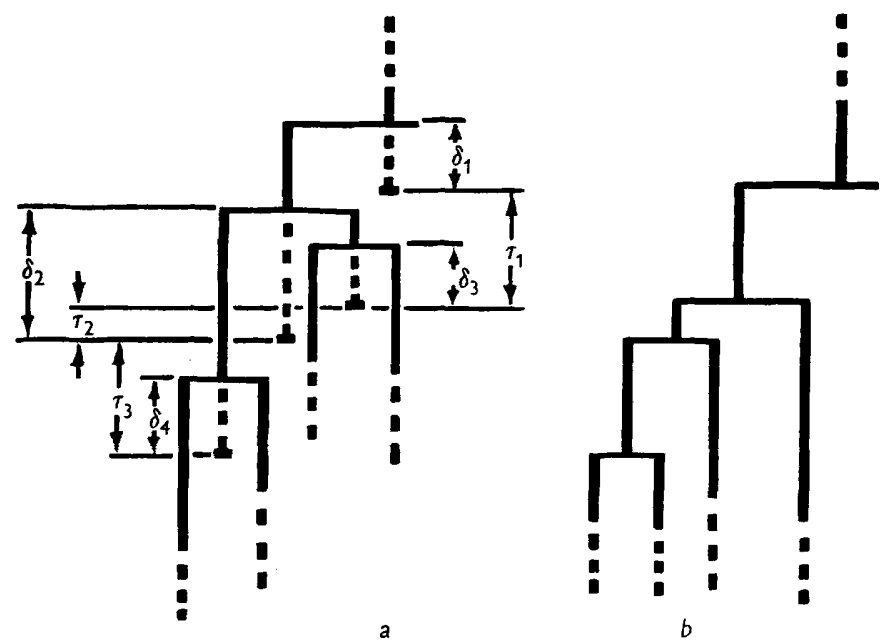

Fig. 5. Relation between the $p$-tree (a) (solid line) and the observed $o$-tree $(b)$ when the $\delta$ are unrestricted.

I think that this explanation of the sign of $\rho_{F}(H)$ is correct in substance; although, regarded as a hypothesis, it is only feebly supported by the data, I can think of no phenomena with which it is in conflict. The formal analogy with the treatment of observational error (Equations (2) and (3)) is exact: var $\delta$ biases the observed $\rho$ towards $+\frac{1}{2}$ or $-\frac{1}{2}$ as the case may be. We have, however, no knowledge of the covariances so summarily omitted, and we have again implicitly assumed that the $\rho_{F}$ are independent of the local growth rate. The chief lesson of this discussion is that even if the $\tau_{p}$ are subject to a law of the Kendall or Rahn type, this fact will not necessarily manifest itself in relations between the $\tau$. It is tempting to pursue the consequences of (9) and (10) further, but such speculation had better wait upon the outcome of more experimental work.

The condition that the $\delta$ shall be less than the corresponding $\tau_{p}$ is essential to the foregoing argument. Fig. $5 a$ represents a $p$-tree with $\delta$ which are not so restricted. The $p$-fissions occur at the beginning of the $\delta$ intervals, the $o$-fissions at their end. The interval $\delta_{2}$ is chosen long enough to overlap $\delta_{3}$ and the preceding $p$-generation time, so that during $\delta_{3}$ the tree represents an organism containing three protoplasts. (Three branches of the $p$-tree coexist with only one of the $o$-tree during $\delta_{3}$.) The successive intervals $\tau_{1}, \tau_{2}, \tau_{3}$ are recorded as 
$o$-generation times, but $\tau_{1}$ corresponds to no single $o$-generation time, and $\tau_{2}$ should be regarded as negative. The observed $o$-tree is shown in Fig. $5 b$. Thus if there is no restriction on the $\delta$ the $o$ - and $p$-trees are not in general homoeomorphic (in the topological sense) and though a unique one-one correspondence between their nodes must still exist, it cannot be inferred by inspection of the $o$-tree alone.

So large an aberration as is represented by $\delta_{2}$ (Fig. $5 a$ ) is perhaps rare in unicellular organisms, but without special techniques it could only be directly recognized in cocci (Powell, 1955).

\section{The status of the coefficient of variation}

The coefficient of variation of generation time has a special significance in the hypotheses of Kendall and Rahn: it is closely related to the number of primitive steps in the postulated mechanism of fission. The relation is, for Kendall's hypothesis,

and for Rahn's

$$
\begin{gathered}
c^{2}=1 / g, \\
c^{2}=\frac{\Gamma_{2}(1)-\Gamma_{2}(1+g)}{\left\{\Gamma_{1}(1+g)-\Gamma_{1}(1)\right\}^{2}},
\end{gathered}
$$

where $g$ is the number of primitive steps, $c$ the coefficient of variation, and

$$
\Gamma_{n}(x)=(d / d x)^{n} \log \Gamma(x) .
$$

Now that the measured generation times are recognized to pertain to secondary processes, the further examination of their coefficients of variation would seem unpromising; it turns out that a qualitative treatment exposes some features of interest.

Consider a single line of descent in a large family tree, and choose a time scale such that the mean $\bar{\tau}$ is unity. A segment of the line of temporal length $n$ might represent about $n \pm \sqrt{ }(n$ var $\tau) o$-generation times, and $n \pm \sqrt{ }\left(n \operatorname{var} \tau_{p}\right)$ $p$-generation times. But these numbers must not in any particular instance differ by more than two units if the morphology of the ancestor (supposed normal) is to be preserved; the $o$-fissions must keep nearly in step with the $p$-fissions, and the $\tau$ and $\tau_{p}$ must both regress (in Galton's sense) towards the same mean $\bar{\tau}=\bar{\tau}_{p}$. If $\tau_{p}$ and $\tau$ (or $\tau_{p}$ and $\delta$ ) were independent variables, it would sometimes happen that a line of descent would contain more $o$-fissions than $p$-fissions; this is impossible, save for irregularity of one unit at the ends of the segment. Equally, the occurrence of more $p$-fissions than $o$-fissions would imply that multicellular individuals had been produced (Fig. 5), and the multicellular condition once attained might be inherited for an indefinite period (cf. Feller, 1950, p. 249 seq.). The observation that the morphology is preserved, with rare individual exceptions, shows that the variations in $\tau$ and $\tau_{p}$ are closely interlinked. Since in units of $\bar{\tau}, \operatorname{var} \tau$ is $c^{2}$, we may expect a rough parallelism between $c^{2}(\tau)$ and $c^{2}\left(\tau_{p}\right)$ though this is of course not a logical necessity.

Incidentally, it is now evident that such covariances as $\operatorname{cov}\left(\tau_{p 1}, \delta_{1}\right)$ in Equation (8) (see Fig. 4) are almost certainly not zero, though they may be 
negligible for the purpose of the argument there developed, which is restricted to local fluctuations.

The observed $c^{2}$ are listed in Table 7. They are all smaller than the $c^{2}$ derived from Kelly \& Rahn (1932) or from my previous data, and imply, without any allowance for inter-experiment variance, that for Kendall's hypothesis (from Equation (11))

$$
g=21 \text { to } 57,
$$

and for Rahn's (from Equation (12))

$$
g=200 \text { to } 8000 \text {. }
$$

Also given in Table 7 are coefficients of variation $c_{0}$ corrected for interexperiment variance by means of Equation (4), $c_{j}$ being taken as $\sigma_{E}^{2} / a^{2}$ (Table 2, A). The true coefficients probably lie between $c$ and $c_{0}$. The $c_{0}^{2}$, which seem to be rather more uniform than the $c^{2}$, then lead to the following revised estimates of the $g$ :

$$
\begin{aligned}
& \text { Kendall's } g=23 \text { to } 93 \text {; } \\
& \text { Rahn's } g=260 \text { to } 130,000 \text {. }
\end{aligned}
$$

These last figures demonstrate the insensitivity of $c^{2}$ (in Equation (12)) to changes in $g$, and the impracticability of determining the constancy of the latter. Still, Rahn's identification of his $g$ with the number of genes in the organism now appears much more acceptable; such rough estimates of the number as can be made on other grounds (Haldane, 1954) fall within the above range. But the fact that the $\tau$, and the high probability that the $\tau_{p}$ of related organisms, are associated deprives the data, in their purely numerical aspect, of all cogency.

For reasons already discussed in connexion with the homogeneity of variance, I have made no formal comparisons of the $c^{2}$, either between series or between experiments; instead, I have carried out two forms of test for stability, the first for independence of the local growth rate, the second for independence, under nominally fixed experimental conditions, of imperfect replication. The first test consisted in forming the correlation coefficient between the mean (say $a_{F}$ ) and the logarithm of the coefficient of variation (say $\log c_{F}$ ) of $F$-hexads, series by series. These two variates are approximately normally distributed. Table $6, \mathbf{A}$ gives the estimated correlation coefficients together with the corresponding $z\left(=\tanh ^{-1} \rho\right)$ and their probabilities. The two extreme values lie outside the 0.05 probability level, but the weighted mean of the $z$ is found to be $+\mathbf{0 . 0 0 2 3}$ - less than its standard error of $\mathbf{0 \cdot 0 3 6}$-and seven of the sixteen are positive. The second test consisted in carrying out a two-way analysis of variance for 'between experiment' and 'within experiment' components of variance of $\log c_{F}$ (see Example 6). The results of this test (Table 6,B) show some excess of the higher $F$-ratios, but only one $P(F)$ is appreciably below 0.05 , and six of the ratios are below unity.

Thus $c_{F}$ is practically independent both of the local growth rate and of minor alterations in the cultural conditions. The coefficient of variation of the whole population will be greater than $c_{F}$ to an extent depending on the 
Table 6.

A : correlation coefficient between mean $\left(a_{F}\right)$ and logarithm of coefficient of variation $\left(c_{F}\right)$ of $F$-hexads, with $z$ and its probability. B: F-ratios (and degrees of freedom) and probabilities for two-way analysis of variance of $\log c_{F}$. (See Example 6.)

A

\begin{tabular}{|c|c|c|c|c|c|c|}
\hline \multirow[b]{2}{*}{ Series } & \multirow[b]{2}{*}{$\rho\left(a_{F}, \log c_{F}\right)$} & \multirow[b]{2}{*}{$\begin{array}{c}\text { No. of } \\
\text { pairs }\end{array}$} & \multirow[b]{2}{*}{$z$} & \multirow[b]{2}{*}{$P(z)$} & \multicolumn{2}{|c|}{ B } \\
\hline & & & & & $F,\left(n_{1}, n_{2}\right)$ & $P(F)$ \\
\hline $\begin{array}{r}\mathrm{Ba} 1 \\
\mathbf{2}\end{array}$ & $\begin{array}{l}-0.053 \\
+0.004\end{array}$ & $\begin{array}{l}60 \\
62\end{array}$ & $\begin{array}{l}-0.053 \\
+0.004\end{array}$ & $\begin{array}{l}0.69 \\
0.96\end{array}$ & $\begin{array}{l}1 \cdot 82(9,50) \\
1.81(8,53)\end{array}$ & $\begin{array}{c}>0.05 \\
c a .0 .1\end{array}$ \\
\hline $\mathbf{3}$ & -0.055 & 30 & -0.055 & 0.77 & $1 \cdot 40(6,23)$ & $>0.25$ \\
\hline 4 & -0.088 & 65 & -0.088 & 0.49 & $0.44(7,57)$ & $>0.1$ \\
\hline 5 & -0.029 & 47 & -0.029 & 0.85 & $2 \cdot 21(8,38)$ & $c a .0 \cdot 05$ \\
\hline 6 & $+0 \cdot 270$ & 60 & +0.277 & 0.04 & $0.72(8,51)$ & $>0 \cdot 1$ \\
\hline Cp 1 & +0.398 & 60 & $+0 \cdot 421$ & 0.00 & $1 \cdot 64(8,51)$ & $>0.1$ \\
\hline 2 & +0.031 & 47 & +0.031 & 0.84 & $2 \cdot 42(7,39)$ & $c a .0 .05$ \\
\hline 3 & $+0 \cdot 182$ & 22 & $+0 \cdot 184$ & 0.42 & $2 \cdot 47(3,18)$ & $c a .0 \cdot 1$ \\
\hline 4 & $-0 \cdot 197$ & 56 & -0.200 & $0 \cdot 14$ & $4.48(8,47)$ & $<0.001$ \\
\hline 5 & -0.234 & 34 & -0.238 & $0 \cdot 16$ & $0.76(6,27)$ & $>0.1$ \\
\hline Sf 1 & $+0 \cdot 151$ & 56 & +0.152 & $0 \cdot 27$ & $1 \cdot 33(8,47)$ & $>0.25$ \\
\hline 2 & +0.091 & 68 & +0.092 & 0.46 & $0.92(9,58)$ & $>0.1$ \\
\hline 3 & $-0 \cdot 066$ & 70 & -0.066 & 0.59 & $0.91(8,61)$ & $>0 \cdot 1$ \\
\hline Pp 1 & -0.375 & 45 & -0.394 & 0.01 & $1 \cdot 88(7,37)$ & $c a .0 \cdot 1$ \\
\hline 2 & $-0 \cdot 161$ & 37 & $-0 \cdot 162$ & 0.32 & $0.25(7,29)$ & $c a .0 .05$ \\
\hline
\end{tabular}

Example 6. Series $S f 3$

2-way analysis of variance of $\log c_{F} ; c_{F}$ the coefficient of variation of $\tau$ in F-hexads

No. of experiments $=9$.

Total no. of $\boldsymbol{F}$-hexads $=70$.

No. of $F$-hexads in individual experiments $=14,12,10,8,6,6,6,4,4$.

$\begin{array}{cccc}\begin{array}{c}\text { Source of } \\ \text { variance }\end{array} & \begin{array}{c}\text { Sum of } \\ \text { squares }\end{array} & \begin{array}{c}\text { Degrees } \\ \text { of freedom }\end{array} & \begin{array}{c}\text { Mean } \\ \text { square }\end{array} \\ \text { Between experiments } & \mathbf{1 \cdot 8 2 7 4} & 8 & \mathbf{0 \cdot 2 2 8 4} \\ \text { Within experiments } & \mathbf{1 5 \cdot 3 8 8 6} & 61 & \mathbf{0 \cdot 2 5 2 3} \\ \text { Total } & \mathbf{1 7 \cdot 2 1 6 0} & 69 & -\end{array}$

$F=0.9053 ; P(F)>0 \cdot 1$.

inter-family variance and determined by an equation of the form (4); it can be constant only if the inter-family variance is so. We do not know if this is the case, but the two tests together suggest that the coefficient of variation may indeed reflect some special feature of the reproductive process.

The two series $\mathrm{Ba} 1$ and $\mathrm{Sf} 1$ which were carried out at a lower temperature than the rest (Table 1) are quite insufficient to show whether or not $c$ (or $c_{0}$ ) varies with temperature; neither pair of values is extreme for the organism concerned (Table 7). Nor is there any clear indication that the chemical complexity of the medium affects $c$ in a systematic way.

In Table 8, the coefficients of variation are classified according to organism and complexity of medium. The media TMB and PW are taken as 'complex' since they offer a variety of amino acids and atomic groupings which would 
Table 7. Coefficients of variation of the raw data $\left(c^{2}\right)$ and corrected for

\begin{tabular}{|c|c|c|}
\hline Series & $c^{2}$ & $c_{0}^{2}$ \\
\hline $\mathrm{Ba} \mathbf{I}$ & 0.0422 & 0.0348 \\
\hline 2 & 0.0451 & $0 \cdot 0399$ \\
\hline 3 & 0.0312 & $0 \cdot 0264$ \\
\hline 4 & $0 \cdot 0485$ & $0 \cdot 0439$ \\
\hline 5 & 0.0287 & $0 \cdot 0258$ \\
\hline 6 & 0.0344 & 0.0266 \\
\hline Cp 1 & $0 \cdot 0446-$ & $0 \cdot 0260$ \\
\hline 2 & 0.0319 & 0.0271 \\
\hline 3 & $0.0446+$ & 0.0323 \\
\hline 4 & 0.0384 & 0.0287 \\
\hline 5 & 0.0276 & 0.0249 \\
\hline Sf 1 & 0.0206 & 0.0134 \\
\hline 2 & 0.0176 & $0 \cdot 0107$ \\
\hline 3 & 0.0277 & 0.0204 \\
\hline Pp 1 & 0.0282 & 0.0216 \\
\hline 2 & 0.0198 & $0 \cdot 0164$ \\
\hline
\end{tabular}

Table 8. Coefficients of variation, uncorrected $\left(c^{2}\right)$ and corrected $\left(c_{0}^{2}\right)$ for apparent inter-experiment variance, classified according to organism and complexity of medium

Organism
Aerobacter cloacae
Serratia marcescens
Pseudomonas aeruginosa

\begin{tabular}{|c|c|}
\hline \multicolumn{2}{|c|}{$1000 c^{2}$} \\
\hline $\begin{array}{c}\text { Complex } \\
\text { media }\end{array}$ & $\begin{array}{l}\text { Simple } \\
\text { media }\end{array}$ \\
\hline 42 & 29 \\
\hline \multirow{3}{*}{45} & 31 \\
\hline & 34 \\
\hline & $48^{*}$ \\
\hline \multirow[t]{4}{*}{$45-$} & 28 \\
\hline & 32 \\
\hline & 38 \\
\hline & $45+*$ \\
\hline 28 & 20 \\
\hline
\end{tabular}

$\begin{array}{cc}\overbrace{\text { Complex }} & 1000 c_{0}^{2} \\ 35 & \begin{array}{c}\text { Simple } \\ \text { media }\end{array} \\ 40 & 26 \\ & 26 \\ & 27 \\ 26 & 44^{*} \\ & 25 \\ & 27 \\ & 29 \\ 22 & 32^{*} \\ & 16\end{array}$

otherwise have to be synthesized by the organism; all the synthetic media are taken as 'simple'. Thirteen comparisons can be made between the $c^{2}$ for complex and the $c^{2}$ for simple media; in ten of these the complex medium gives the larger $c^{2}$. For $c_{0}^{2}$, the corresponding ratio is $8 / 13$. The entries marked with an asterisk in Table 8 correspond to the medium HP (Series $\mathrm{Ba} 4$ and $\mathrm{Cp} 3$ ) containing sodium pyruvate; this medium appears to be somewhat toxic since it increases $\boldsymbol{a}$ and var $\tau$, as well as $c^{2}$, relative to the simpler medium $\mathbf{H}$ (compare series $\mathrm{Ba} 3$ and 4, $\mathrm{Cp} 2$ and 3 in Table 10). If these entries are deleted, ten possible comparisons remain; in all of them $c^{2}$ is greater for the complex medium; for $c_{0}^{2}$, the ratio is $8 / 10$. I do not think that much weight can be given to these latter comparisons; there is no satisfactory definition or criterion of toxicity, and certainly increased generation time alone is not necessarily indicative. There is, then, enough evidence to encourage a further study of the 
effect of medium on $c^{2}$, but no more. It is of the greatest importance that such a study should be undertaken. If it were found that the higher values of $c^{2}$ were systematically associated with the more complex growth media, the fact would constitute strong evidence in favour of a generalized hypothesis of the Kendall type, namely, that the dispersion of generation time is dependent on the number of synthetic processes the organism is called upon to perform in order to convert pabulum into living matter.

\section{The incidence of non-viable organisms}

There are two reasons for introducing this apparently alien subject:

(i) A healthy fully nourished culture produces only a small proportion of non-viable organisms, and it is therefore economical to record their occurrence on any occasion that offers. Very few satisfactory observations have ever been made; the classical methods for determining the proportion of non-viable organisms in a culture are susceptible to many kinds of error, and they are of little value when the proportion is small.

(ii) The fact that non-viable organisms are produced in favourable environments which are chemically and physically uniform gives them a title to inclusion in a description of the pattern of generation times. They can scarcely be ignored, for the effect of including them in the frequency function itself is to make the mean infinite.

It is not possible at present to set up a definition of viability. An adopted criterion must depend on reproductive power, since neither growth nor any chemical activity is uniquely a property of living organisms; furthermore, viability itself is not absolute, but only has a meaning in an environment which can be specified (Powell, 1956 b). I submit the following remarks to provide a definite context for the few data I have to present, and so to avoid misunderstanding. The subject is fraught with a number of grave philosophical difficulties which have never been resolved and of which no mature discussion is yet possible. I believe that they can be resolved, if at all, only by an appeal to detailed observation.

I adopt the following terminology: a bacterium will be said to have been $I$-viable (from 'Individual') in such-and-such an environment if it is known to have undergone fission. This retrospective kind of statement is the only kind that can be made with certainty about the viability of an organism. The assertion of present viability is always doubtful, and of non-viability utterly precarious. (See, for example, Heinmets, Taylor \& Lehman, 1954.) I assume, however, that an organism can meaningfully be said to be $I$-viable with probability $\alpha$ (or $I$-non-viable with probability $1-\alpha$ ) by induction from previous or contemporary experience. The state of an organism may be determinate, in which case $\alpha$ is merely a sampling frequency, or a chance element may enter the biological process itself; this is a matter for experiment to decide.

A knowledge of the viability of the individual in the above sense, i.e. of its capacity to undergo fission, is often of little interest to the biologist. More usually what is wanted is a knowledge of the probability that it will give rise 
to a large number of progeny after many generations. (Compare the discussion in Valentine $\&$ Bradfield, 1954.) I shall say that an organism has been $S$-viable (from 'Species') if it has given rise to an indefinitely long sequence of generations of progeny in such-and-such a constant environment. The uncertainty attached to the expression 'indefinitely long' could be diminished by a convention, but in many cases there is no practical objection to it. Suppose that in a culture growing under constant conditions a fraction $\alpha$ of the organisms which appear are $I$-viable, and that the occurrences of $I$-non-viable organisms are all independent of one another. Then if $\alpha \leqslant \frac{1}{2}$, the culture will die out. If $\alpha>\frac{1}{2}$, every $I$-viable organism is potentially $S$-viable, and it can properly be said that the species is viable in that environment. Feller (1950) proves a result equivalent to the following: when $1>\alpha>\frac{1}{2}$ the number of $n$th generation progeny of an $I$-viable ancestor tends to infinity with $n$, or is 0 , except in a vanishingly small proportion of cases. Under the assumptions of this example, the distinction between $S$-viability and $S$-non-viability is sharp: unless $\alpha$ differs by very little from $\frac{1}{2}$, an organism can be classified with a close approach to certainty after only a few generations of growth. Here also the relation between $I$-viability and $S$-viability is simple: the probability, $\beta$, that an $I$-viable organism will also be $S$-viable is given by

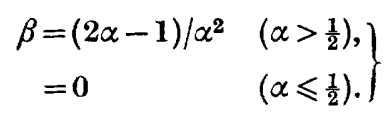

There are definite reasons why attention should first be fixed on the property of $I$-viability: (i) it appears to be more elementary than $S$-viability; (ii) it is easier, in working under the microscope, to establish constant and repeatable conditions for a short time than for a long time; (iii) given the laws of incidence of $I$-viability, those of $S$-viability can be inferred; the converse is in general false.

Henceforward I use 'viable' to mean $I$-viable. In the present context I call an organism non-viable (i) if it becomes only dimly visible under phase-contrast illumination and then shows no sign of growth during about $1 \mathrm{hr}$.; (ii) failing (i), if it shows no sign of growth during about $3 \mathrm{hr}$.

The experiments provide a sample of $\mathbf{3 4 5 5}$ fissions, actually seen or known to have occurred in the culture chamber, and such that the fate of both organisms stemming from each is known. These are classified in Table 9. The index of viability, $\alpha$, i.e. the proportion of viable organisms in the sample, is calculated for each species ( $\alpha$ is one-half of the 'generation index', $p$, of Topley \& Wilson's Principles, 1955). The number of non-viable organisms is too small to disclose any significant difference between species, still less between series; the grand total gives $\alpha=0.994$ : among newly formed organisms probably less than $1 \%$ are non-viable.

The index $\alpha$ is not the same as the proportion of viable organisms in a growing culture. In steady exponential growth the proportion tends, as the effect of the constitution of the inoculum is diluted out, to $2 \alpha-1$ (see, for example, Topley \& Wilson's Principles, 1955). The same value obtains in continuous cultures of the Monod (1950) type (Powell, 1956c). Here

$$
2 \alpha-1=0.988 \text {, }
$$


in good agreement with the value 0.984 obtained by Valentine \& Bradfield (1954). Much lower values obtained by classical methods (e.g. 0.90; Wilson, 1922) have been widely accepted, but my own desultory experience with a few representatives of the genera Bacillus, Mycobacterium, Corynebacterium, Staphylococcus, Salmonella and Pasteurella suggests that $\alpha$ is very generally near 0.99 in unrestricted growth.

\section{Table 9. Incidence of non-viable organisms}

The index of viability $\alpha$ is the proportion of viable organisms in the sample.

\begin{tabular}{|c|c|c|c|c|}
\hline \multirow[b]{2}{*}{ Series } & \multicolumn{4}{|c|}{ No. of fissions giving rise to } \\
\hline & $\begin{array}{l}\text { Total } \\
\text { no. of } \\
\text { flissions }\end{array}$ & $\begin{array}{l}1 \text { non-viable } \\
\text { organism }\end{array}$ & $\begin{array}{c}2 \text { non-viable } \\
\text { organisms }\end{array}$ & $\alpha$ \\
\hline $\mathrm{Ba} 1$ & 199 & 1 & -1 & \\
\hline 2 & 194 & 2 & - & \\
\hline 3 & 183 & - & 2 & 0.0950 \\
\hline 4 & 222 & 2 & - & 0.9900 \\
\hline 5 & 189 & 1 & - & \\
\hline 6 & 213 & 2 & - & \\
\hline Cp 1 & 196 & 3 & -1 & \\
\hline 2 & 217 & - & - & \\
\hline 3 & 260 & 5 & 1 & 0.9945 \\
\hline 4 & 220 & 1 & - & \\
\hline 5 & 205 & 1 & $-J$ & \\
\hline Sf 1 & 212 & 4 & 2) & \\
\hline 2 & 262 & 2 & $-\}$ & 0.9925 \\
\hline 3 & 255 & 1 & $-)$ & \\
\hline Pp 1 & 217 & 1 & $2\}$ & \\
\hline 2 & 211 & 2 & $-\}$ & 0.9918 \\
\hline Totals & 3455 & 28 & 7 & 0.9939 \\
\hline
\end{tabular}

If we classify fissions as $V V, N V$ and $N N$ according as they produce none, one, or two non-viable organisms, we have for the expected proportions of these types

$$
V V: N V: N N=\alpha^{2}: 2 \alpha(1-\alpha):(1-\alpha)^{2},
$$

or, in a sample of 3455 with $\alpha=0.9939$,

$$
V V: N V: N N=3413 \cdot 13: 41 \cdot 74: 0 \cdot 13 .
$$

The numbers observed are

$$
V V: N V: N N=3420: 28: 7 .
$$

The expected number of type $N N$ is too small to permit the use of a $\chi^{2}$ test. Instead, we may reason thus : the expected number of type $N N$ is so small that its sampling distribution will be approximately Poissonian. The standard error of $1-\alpha$ (42 of 6910 observations) is about $0.001-$ one-sixth of its value. Hence with great confidence we can say that the true expectation of $N N$ is below 1 , since $3455(1-\alpha)^{2}$ is only $0 \cdot 13$. But the chance of 7 or more in a Poisson distribution with parameter 1 is about 0.0006 . Thus there is a strong indication that the viabilities of sisters are positively associated. 
Of the 35 fissions giving rise to non-viable organisms (Table 9), 16 are the terminations of organisms of known generation time. Now the generation time of an organism one or both of whose progeny are non-viable will lie as often above as below the median of the $\tau$ distribution, if it is independent of the fate of the progeny. Allowing for possible inter-experiment variance, we should compare the $\tau$ of such organisms with the median $\tau$ of the experiment in which they occurred. It turns out that in 14 of the 16 available instances, the $\tau$ of the mothers lie above the experiment means and a fortiori (since the distributions are positively skew) above the medians. The probability of this occurrence is about 0.008 on the hypothesis of independence. It appears that a falling growth rate may precede the state of complete incompetence. The corresponding result for the viable sisters of non-viable organisms is neither striking nor significant (14 of 21 instances).

The most obvious way in which the incidence of non-viable organisms fails of being purely fortuitous is through the association between the fates of sisters. This phenomenon leads to a relation different from Equation (13) for the connexion between $I$-viability and $S$-viability. It is easy to show that, if $\beta_{n}$ is the probability that an $I$-viable organism has $I$-viable progeny of the $n$th generation,

$$
1-\beta_{n}=(1-\alpha)^{2}+2 \alpha(1-\alpha)\left(1-\beta_{n-1}\right)+\alpha^{2}\left(1-\beta_{n-1}\right)^{2},
$$

where $\alpha$ is the index of viability, supposed constant, and where the occurrences of $I$-non-viability are supposed independent of one another. In the limit, as $n \rightarrow \infty$, (14) reduces to (13), giving the probability, $\beta$, that an $I$-viable organism is also $S$-viable. In (14) the coefficients $(1-\alpha)^{2}, 2 \alpha(1-\alpha), \alpha^{2}$ are the probabilities that both sisters of a pair are $I$-non-viable, that only one is so, and that neither is so, respectively. Hence we may write the limiting form of (14) more generally as

$$
1-\beta=C+2 B(1-\beta)+A(1-\beta)^{2},
$$

where now $A C \neq B^{2}$ if the fates of sisters are not independent. A convenient measure of association is Yule's (1912) coefficient of absolute association, $V$, which is closely related to $\chi^{2}$ in a $2 \times 2$ contingency table:

$$
V=\frac{A C-B^{2}}{(A+B)(B+C)} .
$$

$$
\begin{aligned}
& A+2 B+C=1 \text {, } \\
& A+B=\alpha, \\
& A=\alpha^{2}+\alpha(1-\alpha) V, \\
& B=\alpha(1-\alpha)(1-V), \\
& C=(1-\alpha)^{2}+\alpha(1-\alpha) V \text {, }
\end{aligned}
$$

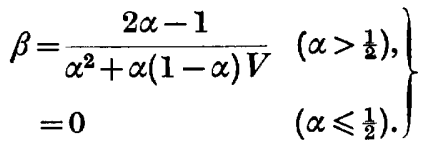


So the effect of positive association between sisters is to reduce the probability that an $I$-viable organism is also $S$-viable. For the totals of Table $9, V$ is 0.329 , and from (16), with $\alpha=0.9939, \beta=0.998$.

When $V=0$ (Equation 13)

$$
\beta=1 \cdot 000-.
$$

Only if $\alpha$ is less than about 0.9 will $\beta$ be practically distinguishable from unity, whatever $V$ is.

These considerations may be of some importance in the conventional method of determining a viable count by 'plating-out'. The method determines the number of organisms in the sample which are $S$-viable under the conditions of the test. Of course equations such as (13) or (16) are not likely to be obeyed exactly, because of the erratic growth of organisms on transfer to a new environment, and because in ordinary practice the supply of nutrients is not held constant; but the distinction between $S$-viability and $I$-viability and the probable change in $\alpha$ and $\beta$ in the new environment ought to be remembered whenever it is known or suspected that $\alpha<0 \cdot 9$.

\section{The distribution of generation times}

In the absence of a satisfactory working hypothesis, and in view of the uncertainty about inter-experiment variance, the data may as well be represented by any frequency function which fits adequately and which is practically convenient. In the choice of suitable functions $I$ have been guided by the increasing importance of 'continuous culture' as a method of studying growth and of conducting large-scale fermentations. A knowledge of the generation time distribution is of great value in understanding the behaviour of such cultures (Powell, 1956c). Through this section I use $f(\tau)$ as a general notation for a frequency function of generation times, exclusive of non-viable organisms. Another, derived, generation time distribution, $\mathscr{C}(\tau)$ which $I$ have called the 'carrier distribution' is important in determining the selective properties of continuous cultures. In the absence of appreciable mother-daughter correlation, and neglecting the small proportion of non-viable organisms,

$$
\mathscr{C}(\tau) \equiv 2 e^{-\nu \tau} f(\tau),
$$

where $\nu$ is the number growth-rate constant, defined by

$$
\int_{0}^{\infty} e^{-\nu \tau} f(\tau) d \tau=\frac{1}{2}
$$

Equation (17) limits the forms of $f(\tau)$ which can be conveniently handled to those having simple characteristic functions (or Laplace transforms); it excludes the Pearson Type VI and the otherwise attractive 'lognormal' distributions. Of the familiar distributions which are also singly terminated there remain the Pearson types III:

and V:

$$
\begin{aligned}
& f(\tau) \equiv \frac{\tau^{g-1} e^{-\tau / m}}{m^{g} \Gamma(g)}, \\
& f(\tau) \equiv \frac{m^{g} \tau^{-g-1} e^{-m / \tau}}{\Gamma(g)} .
\end{aligned}
$$


A possible alternative to the Pearson Type VI is the three-parameter distribution

$$
f(\tau) \equiv \frac{e^{-\tau / m}\left(1-e^{-\tau / \lambda m}\right)^{g-1}}{\lambda m B(\lambda, g)},
$$

where $B(\lambda, g)$ is the 'complete' beta-function. (The genesis of Equation (20), which is a generalization of Yule's (1925) distribution is discussed by Jensen (1954); it is a member of the class of distributions considered by Kendall \& Waugh (Kendall, 1952), namely those in which the variate is the sum of $g$ variates each distributed as a negative exponential of, in general, arbitrary parameter. I am indebted to Dr D. G. Kendall for the Jensen reference.) When Equation (17) holds, Equations (18) and (20) evidently give rise to carrier distributions of the same functional form as themselves and the growthrate constants are readily found to be given by:

Pearson Type III: $\quad \quad \quad=\frac{1}{m}\left(2^{1 / g}-1\right) ;$

Distribution (20): $\quad \frac{\mathbf{1}}{\mathbf{2}}=\frac{\Gamma\{\lambda(1+m \nu)\} \Gamma(\lambda+g)}{\Gamma\{\lambda(1+m \nu)+g\} \Gamma(\lambda)}$.

For the Pearson Type V we have

$$
1=\frac{2}{\Gamma(g)} \int_{0}^{\infty} m^{g} \tau^{-g-1} \exp (-\nu \tau-m / \tau) d \tau .
$$

On putting $a=2 \sqrt{(m \nu),} b=\sqrt{ }(m / \nu), \tau=b e^{\xi}$ and rearranging:

$$
1=\frac{4 m^{g}}{b^{g} \Gamma(g)} \int_{0}^{\infty} e^{-a \cosh \xi} \cosh g \xi d \xi,
$$

whence

$$
4 m^{g / 2} \nu^{g / 2} K_{g}\{2 \sqrt{ }(m \nu)\}=\Gamma(g),
$$

where $K_{g}(a)$ is the modified Bessel function of the second kind (with the definition adopted by Watson (1952)).

Table 10 gives the estimates of the first $\left(\mu_{1}^{\prime},=a\right)$ and second $\left(\mu_{2},=\operatorname{var} \tau\right)$ moments and the skewness $\left(\gamma_{1},=\sqrt{ } \beta_{1}\right)$ and kurtosis $\left(\gamma_{2},=\beta_{2}-3\right)$ of the observations. The representative points of the distributions in the Pearsonian $\beta_{1}, \beta_{2}$ diagram (see, for example, Pearson \& Hartley, 1954) are widely scattered; many fall near the Type III and Type $V$ lines, but a few are far away in the Type IV area. I have not attempted to fit Type IV or Type VI distributions for the reason given above. Furthermore, the moments of some of the series can be accommodated to a Type VI only if its lower terminus lies above the origin. The arithmetic and geometric means for the Type III, and the harmonic and geometric means for the Type V, are sufficient statistics for the parameters $g, m$ when the origin is taken as the lower terminus. The results of fitting both frequency functions by the method of maximum likelihood are given in Table 11. In only three series do both give a $\chi^{2}$ probability below $\mathbf{0} \cdot 05$. We may take it that the distribution of $\tau$ can be represented for practical 
purposes by either a Type III or a Type V, though the $\chi^{2}$ totals show that neither alone is satisfactory throughout. This conclusion is strengthened by the existence of association between the generation times of related organisms. If the $\tau$ of an organism falls in a given group, that of its sister is more likely to fall in the same group than it would be in the absence of association, and the true expectation of $\chi^{2}$ is rather greater than $n$, the number of degrees of freedom.

Table 10. Estimates of the first $\left(\mu_{1}^{\prime},=a\right)$ and second $\left(\mu_{2},=\right.$ var $\left.\tau\right)$ moments and the skewness $\left(\gamma_{1}\right)$ and kurtosis $\left(\gamma_{2}\right)$ of the crude $\tau$-distributions

\begin{tabular}{|c|c|c|c|c|c|}
\hline \multirow[b]{2}{*}{ Series } & \multirow[b]{2}{*}{$\begin{array}{c}\text { No. of } \\
\text { observations }\end{array}$} & \multicolumn{2}{|c|}{ The unit of time is $1 \mathrm{~min}$} & \multirow[b]{2}{*}{$\gamma_{1}$} & \multirow[b]{2}{*}{$\gamma_{2}$} \\
\hline & & $\mu_{1}^{\prime}$ & $\mu_{2}$ & & \\
\hline Ba 1 & 372 & $29 \cdot 57$ & 36.9 & 0.81 & 2.5 \\
\hline 2 & 372 & $24 \cdot 42$ & $26 \cdot 9$ & $0 \cdot 38$ & 1.7 \\
\hline 3 & 318 & $49 \cdot 15$ & 75.5 & 0.65 & 0.9 \\
\hline 4 & 394 & $52 \cdot 35$ & 132.8 & 1.31 & 4.9 \\
\hline 5 & 323 & $27 \cdot 51$ & $21 \cdot 6$ & 0.55 & $0 \cdot 3$ \\
\hline 6 & 378 & $33 \cdot 74$ & $39 \cdot 2$ & 0.95 & $1 \cdot 3$ \\
\hline Cp 1 & 367 & $22 \cdot 88$ & $23 \cdot 3$ & 1.61 & 6.7 \\
\hline 2 & 377 & $81 \cdot 84$ & $213 \cdot 4$ & 0.68 & $1 \cdot 0$ \\
\hline 3 & 376 & 86.06 & $330 \cdot 4$ & 0.62 & $0 \cdot 2$ \\
\hline 4 & 398 & 53.59 & $110 \cdot 3$ & 0.84 & 2.9 \\
\hline 5 & 319 & $75 \cdot 66$ & $157 \cdot 8$ & $0 \cdot 11$ & -0.2 \\
\hline Sf 1 & 364 & $30 \cdot 21$ & 18.8 & 0.83 & $1 \cdot 1$ \\
\hline 2 & 442 & $26 \cdot 12$ & $12 \cdot 0$ & 0.27 & 0.2 \\
\hline 3 & 446 & $27 \cdot 21$ & $20 \cdot 5$ & 1.21 & $8 \cdot 1$ \\
\hline $\mathbf{P p} 1$ & 365 & 31.08 & $27 \cdot 3$ & 0.72 & 1.4 \\
\hline 2 & 329 & $38 \cdot 11$ & 28.7 & $0 \cdot 39$ & $-0 \cdot 1$ \\
\hline
\end{tabular}

Table 11. Frequency function parameters and goodness of fit, by maximum likelihood

Type III (Equation 18)

\begin{tabular}{|c|c|c|c|c|c|c|c|c|c|c|}
\hline \multirow[b]{2}{*}{ Series } & & & & & & & \\
\hline & $g$ & $m$ & $x^{2}$ & $n$ & $P\left(\chi^{2}\right)$ & $g$ & $m$ & $\chi^{2}$ & $n$ & $P\left(\chi^{2}\right)$ \\
\hline Ba 1 & $24 \cdot 2$ & $1 \cdot 22$ & $3 \cdot 85$ & 11 & 0.97 & 23.9 & 678 & $14 \cdot 3$ & 11 & 0.22 \\
\hline 2 & $20 \cdot 8$ & $1 \cdot 18$ & $28 \cdot 9$ & 10 & 0.0013 & $18 \cdot 0$ & 417 & $53 \cdot 4$ & 11 & 0.0000 \\
\hline 3 & $32 \cdot 0$ & $1 \cdot 53$ & $3 \cdot 08$ & 5 & $0 \cdot 69$ & $32 \cdot 2$ & 1534 & $4 \cdot 04$ & 5 & 0.54 \\
\hline 4 & $23 \cdot 0$ & $\mathbf{2 \cdot 2 8}$ & $9 \cdot 82$ & 8 & $0 \cdot 28$ & $22 \cdot 5$ & 1124 & $15 \cdot 6$ & 8 & 0.049 \\
\hline 5 & $\mathbf{3 5} \cdot \mathbf{3}$ & 0.779 & $6 \cdot 39$ & 8 & 0.60 & $35 \cdot 6$ & 952 & $5 \cdot 48$ & 8 & 0.71 \\
\hline 6 & $30 \cdot 7$ & $1 \cdot 10$ & $22 \cdot 9$ & 11 & 0.018 & $32 \cdot 0$ & 1046 & $9 \cdot 99$ & 11 & 0.53 \\
\hline Cp 1 & $24 \cdot 6$ & 0.929 & $20 \cdot 8$ & 8 & 0.0077 & $25 \cdot 9$ & 569 & $15 \cdot 3$ & 9 & $0 \cdot 082$ \\
\hline 2 & $32 \cdot 0$ & $2 \cdot 55$ & $7 \cdot 85$ & 10 & $0 \cdot 64$ & $32 \cdot 1$ & 2544 & $6 \cdot 87$ & 11 & $0 \cdot 81$ \\
\hline 3 & $23 \cdot 0$ & $3 \cdot 75$ & $24 \cdot 1$ & 13 & 0.030 & $23 \cdot 0$ & 1898 & $18 \cdot 3$ & 12 & $0 \cdot 11$ \\
\hline 4 & $26 \cdot 2$ & $2 \cdot 04$ & $19 \cdot 7$ & 7 & 0.0062 & $25 \cdot 5$ & 1313 & $21 \cdot 2$ & 8 & 0.0067 \\
\hline 5 & $35 \cdot 0$ & $2 \cdot 16$ & $32 \cdot 6$ & 10 & 0.0003 & $33 \cdot 6$ & 2467 & $17 \cdot 6$ & $\boldsymbol{9}$ & $0 \cdot 041$ \\
\hline Sf 1 & $49 \cdot 9$ & $0 \cdot 605$ & $20 \cdot 5$ & 7 & 0.0047 & $51 \cdot 3$ & 1521 & $13 \cdot 4$ & 8 & 0.099 \\
\hline 2 & $55 \cdot 2$ & 0.473 & $7 \cdot 87$ & 6 & 0.25 & $54 \cdot 5$ & 1397 & $5 \cdot 26$ & 6 & 0.51 \\
\hline 3 & $36 \cdot 8$ & $0 \cdot 739$ & $11 \cdot 90$ & 8 & $0 \cdot 16$ & $35 \cdot 7$ & 945 & $21 \cdot 2$ & 9 & 0.012 \\
\hline Pp 1 & $36 \cdot 3$ & 0.857 & $8 \cdot 74$ & 10 & $0 \cdot 56$ & $36 \cdot 8$ & 1114 & $7 \cdot 99$ & 9 & 0.54 \\
\hline 2 & $50 \cdot 6$ & 0.753 & $13 \cdot 24$ & 9 & $0 \cdot 15$ & $50 \cdot 8$ & 1898 & $10 \cdot 7$ & 10 & 0.38 \\
\hline Totals & - & - & $242 \cdot 2$ & 141 & 0.0000 & - & - & $240 \cdot 6$ & 145 & 0.0000 \\
\hline
\end{tabular}


In a few series I have also fitted the two distributions by the method of moments, with results not sensibly different from those given by the method of maximum likelihood. The labour of fitting (20) by the method of maximum likelihood is prohibitive; the moment fit, in the few cases tried, has been less good than that of Type III, the fitted curves being noticeably too unsymmetrical near the mode (Fig. 6).

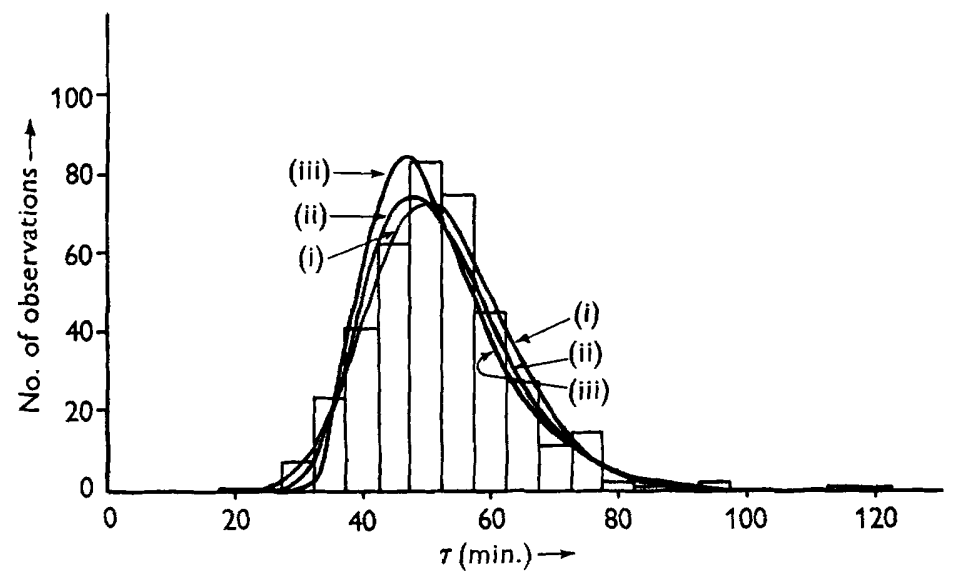

Fig. 6. Distribution of $\tau$ in Series Ba 4. Curve (i) Pearson Type III distribution fitted by maximum likelihood; (ii) Pearson Type V distribution fitted by maximum likelihood; (iii) Generalized Yule distribution (Equation 20) fitted by moments.

\section{CONCLUSION}

In broadest outline, the conclusion is very simple; there is positive association between the generation times of organisms which are closely related by descent. Where the association is unexpectedly weak, i.e. between mothers and daughters, a plausible explanation can be found by an examination of known features of the fission process. This kind of pattern is of course a commonplace in biology; the point of interest is that even among simple organisms chance alone does not determine the individual life-span. And some other properties of bacteria appear to be associated in the same way.

Eddy \& Hinshelwood (1953) exposed washed cells of 'Bacterium lactis aerogenes' to the action of toxic substances (acid, $m$-cresol, acriflavine). They found that the organisms which happened to survive the treatment did not transmit their apparent resistance to their distant progeny; when the progeny were challenged in the same way, the proportion of survivors was the same as in the original culture. I have repeated some of these experiments under the microscope. It would not be appropriate to give details here, but the following result is typical: spores of Bacillus cereus were inoculated on to a culture chamber (Harris \& Powell, 1951) and allowed to grow for $2.5 \mathrm{hr}$. Then a field was selected containing several groups of organisms, each group comprising the progeny of a single spore. The medium was exchanged for a glycine + hydrochloric acid buffer $(\mathrm{pH} \mathrm{3})$ and after $5 \mathrm{~min}$. the original medium was 
replaced. Most of the organisms were destroyed by the acid reagent; the survivors were identified by their resumption of growth:

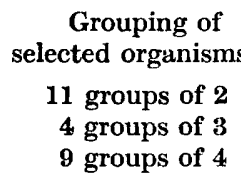

\author{
Survivors \\ 2 organisms in 1 group \\ None \\ 4, organisms in 1 group
}

A few experiments of this kind gave overwhelming evidence for association between sisters and cousins in respect of their resistance to the reagent; but the resistance is not propagated even through one overnight subculture. Similarly Hughes (1955a) found association in the sensitivity of related organisms towards antibiotics, though his object was to demonstrate differences rather than similarities.

There are indications that casual variations in some properties of bacteria may be inherited for many generations, certainly for more than the three covered in my experiments (e.g. Rogers, 1953; Hughes, 1955b). Hughes purports to show that the growth rate is so inherited. He isolated single cells from large and small colonies of Escherichia coli, and allowed them to grow for a fixed time. He found a high correlation between the size of the colonies so formed and the size of those from which the parent cells were isolated. This study, as it is reported, does not prove that the growth rate (or generation time) is inherited in the sense asserted, for two reasons. (i) Inheritance of mean size of organism could be responsible for at least part of the effect. The difference in size of single individuals would not necessarily be obvious under the microscope. (ii) Because growth is a multiplicative process, and the size of a colony doubles during a mean generation time, the size after a given period of growth from a single ancestor is critically dependent on the epoch of fission of the ancestor, and therefore on its age at the beginning of the growth period, and on any lag induced by the manipulations.

I think that Hughes's results do probably demonstrate the persistent inheritance of some property, but it is not necessarily the growth rate. Again, the variety of Aerobacter cloacae which $I$ have used occurs in two forms, one of which has a thicker mucous coat than the other. Both are to be found in ordinary cultures, and single cells of either may transmit their character through ten or more generations, after which reversions to the other form become frequent.

We are, I think, now justified in seeking the beginnings of a working hypothesis to explain the variability of individuals. No other class of organisms offers nearly so good a chance of solving this universal problem.

'An organism tends to keep its equilibrium amid the bombardment of aimless forces which beset it, to regain equilibrium when it has been disturbed, and to pursue a sequence of actions dictated by the need of keeping all its interdependent parts constantly renewed, their structure intact.' (Langer, 1953). The very general phenomenon of variability with regression has been given verbal expression in many ways; for bacteria, Langer's is precisely apt, 
because to them Brownian motion-' the bombardment of aimless forces'-is an immediate and effective reality.

A unicellular organism may contain $10^{10}$ or $10^{11}$ atoms, and of these a quite small fraction will be arranged in specialized ways to form molecules of catalysts or growth factors. Some few will be atoms of heavy metals, essential but required in only exiguous amounts. Thus Haldane (1954) quotes values of about 10 molecules/sec. for the rate of synthesis of some growth factors; the complement of catalase in most bacteria includes $10^{4}$ iron atoms or less (Herbert \& Pinsent, 1948); and the minute amount of molybdenum (perhaps 100 atoms/ cell) necessary and sufficient to enable Azotobacter chroococcum to assimilate elementary nitrogen is well known. The visible Brownian motion of a bacterium suspended in a liquid medium is mostly caused by water molecules, and it is therefore evident that the acquisition of rare atoms or molecules from the medium must be a highly erratic process. Their internal chemistry, too, cannot but be equally erratic. The fewer the atoms or molecules concerned, the greater the relative fluctuation in their number; the slower the reactions in which they are built up and broken down, the more persistent and widespread the effects of that fluctuation. The passage of molecules into the cell, and their reactions with others already present, will be subject to chance effects of two kinds, the first depending on their position in space (becoming diffusion on the large scale), the second on their orientation and thermal energy at critical moments (becoming reaction velocity on the large scale). Transient deviations from the mean rates and concentrations will be frequent, and no doubt sometimes so large as to be reflected in the subsequent behaviour of the organism because they overtax the self-regulating mechanisms, or alter the response to an inimical environment. Occasionally they may be lethal. But we can well admit that the organism is not entirely at the mercy of molecular accidents, and that so relatively simple a creature may be able to resort to more than one stable or metastable state if it is driven to do so. Eddy (1953b), discussing the work of Eddy (1953a) and Eddy \& Hinshelwood (1953) on the survival of organisms treated with acid, adumbrates this same idea; he writes of 'a chance conjunction of events', 'complex fluctuations in the concentrations of many molecular species', as determining the fate of particular individuals. We may perhaps hope in the future to analyse the determinants of generation time fluctuation, or at least to distinguish two in a quantitative way: (i) the immediate chance effect resulting from molecular accidents, as in the hypotheses of Kendall and Rahn; (ii) the inherited tendency whose primary cause may again be accident, but which in any case derives from ancestors more or less remote.

Mr E. C. Fieller (private communication) has suggested the possibility of representing the correlation between generation times as resulting from a process of 'autoregression'. If $\tau_{1}, \tau_{2}$ are generation times of mother and daughter, for example, we might write

$$
\tau_{2}=a \tau_{1}+b x
$$

where $a$ and $b$ are constants, $a+b=1$, and $x$ is a random variable. This scheme, or some elaboration of it, could evidently be made to bear a close analogy to 
the ideas considered above. In its simplest form (21), it implies that the mother-daughter correlation $\rho(H)$ is $a$, and the grandmother-granddaughter (say $\rho\left(H_{2}\right)$ ) is $a^{2}$. It would not be difficult to test the hypothesis that

$$
\rho\left(H_{2}\right)=\{\rho(H)\}^{2} .
$$

In this paper I have treated the observed $\tau$ as representative of a secondary process, keeping in mind the scheme of relations of Figs. 1, 4 and 5. I have already given reasons for the legitimacy of this viewpoint, but at best they should only be taken to apply to unicellular organisms. Consider a $p$-tree as in Fig. 4 or Fig. 5 with associated $\delta$ of small variance but of mean value several times the mean $\tau_{p}$, say about $n \tau_{p}$. Then the 'organisms' produced at $o$-fission will consist of about $2^{n}$ cells, which will grow to about $2^{n+1}$ before the next $o$-fission. We can regard multicellular organisms as being formed in this way. But, remembering the additive property of independent variances, it is clear that even a moderately regular morphology implies close coupling of the $\tau_{p}$ and $\delta$ processes. If, furthermore, the groups of cells are indeed organisms sensu stricto, their $\tau$ will have a biological significance independent of the $\delta$ or of any 'corresponding' $\tau_{p}$. I think therefore that the generation times of multicellular organisms are as much deserving of study as the those of unicellular, especially as our knowledge of intercellular organisation is so little developed.

Mr T. W. Pearce and Mr J. M. Scott assisted in the maintenance of cultures and of the apparatus, and shared in the observations. Much of the computation was carried out by Mr R. Ash.

\section{REFERENCES}

Bisset, K. A. (1955). The Cytology and Life-History of Bacteria, 2nd ed. Edinburgh: E. and $\mathbf{S}$. Livingstone.

Box, G. E. P. (1953). Non-normality and tests on variances. Biometrika, 40, 318.

Daglex, S. \& Hinshelwood, C. N. (1938). Physio-chemical aspects of bacterial growth. Part I. Dependence of growth of Bact. lactis aerogenes on concentration of medium. J. chem. Soc. 1930.

EDDY, A. A. (1953a). Death rate of populations of Bact. lactis aerogenes. II. Environmental and other factors influencing the form of the survival curve. Proc. Roy. Soc. B, 141, 126.

EdDy, A. A. $(1953 b)$. Death rate of populations of Bact. lactis aerogenes. III. Interpretation of survival curves. Proc. Roy. Soc. B, 141, 137.

Eddy, A. A. \& Hinshelwood, C. N. (1953). Death-rate of population of Bact. lactis aerogenes. I. Active adjustment of cells to adverse environments. Proc. Roy. Soc. B, 141, 118.

Feller, W. (1950). Probability Theory and Its Applications. New York: John Wiley and Sons.

HaLdane, J. B. S. (1954). The Biochemistry of Genetics. London: Allen and Unwin Ltd.

HARRis, N. K. \& Powell, E. O. (1951). A culture chamber for the microscopical study of living bacteria, with some observations on the spore-bearing aerobes. J. R. micr. Soc. 71, 407.

Heinmets, F., Taylor, W. W. \& Lehman, J. J. (1954). The use of metabolites in the restoration of the viability of heat and chemically-inactivated Escherichia coli. J. Bact. 67, 5. 

Journal of General Microbiology, Vol. 18, No.2

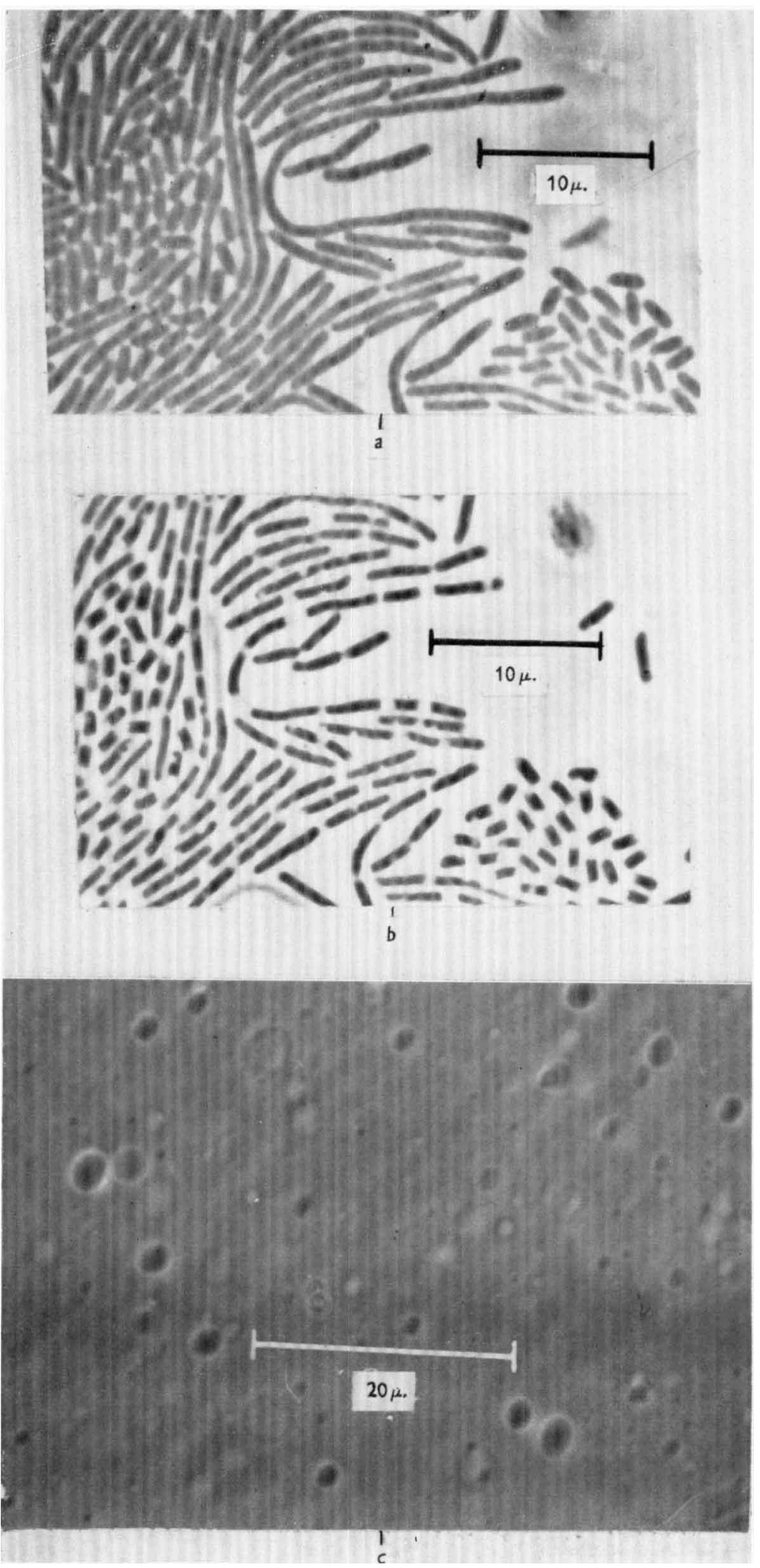

E. O. Powell-The pattern of bacterial generation times. Plate 1

(Facing p. 417) 
Herbert, D. \& Pinsent, J. (1948). Crystalline bacterial catalase. Biochem. J. 43, 193.

Hugres, W. H. (1955 a). The differences in antibiotic sensitivity of closely related single cells of Proteus vulgaris. J. gen. Microbiol. 12, 269.

Hughes, W. H. $(1955 b)$. The inheritance of differences in growth rate in Escherichia coli. J. gen. Microbiol. 12, 265.

Jensen, A. (1954). A Distribution Model. Copenhagen: Munksgaard.

Kelly, C. D. \& RaHN, O. (1932). The growth rate of individual bacterial cells. J. Bact. 23, 147.

KeNDALL, D. G. (1948). On the rôle of a variable generation time in the development of a stochastic birth process. Biometrika, 35, 316.

KeNDaLL, D. G. (1952). On the choice of a mathematical model to represent normal bacterial growth. $J$. $R$. statist. Soc. B, 14, 41.

Langer, S. K. (1953). Feeling and Form. London: Routledge and Kegan Paul.

Monod, J. (1950). La technique de culture continue, théorie et applications. Ann. Inst. Pasteur, 79, 390.

National Bureau of Standards (1949). Tables of the Binomial Probability Distribution. Washington, D.C.: U.S. Government Printing Office.

Pearson, E. S. \& Hartley, H. O. (1954). Biometrika Tables for Statisticians, vol. I. Cambridge University Press.

Powell, E. O. (1955). Some features of the generation times of individual bacteria. Biometrika, 42, 16.

Powell, E. O. (1956a). An improved culture chamber for the study of living bacteria. J. R. micr. Soc. 75, 235.

Powell, E. O. $(1956 b)$. A rapid method for determining the proportion of viable bacteria in a culture. J. gen. Microbiol. 14, 153.

Powell, E. O. $(1956 c)$. Growth rate and generation time of bacteria, with special reference to continuous culture. J. gen. Microbiol. 15, 492.

$\mathbf{R}_{\mathrm{AHN}}$, O. (1932). A chemical explanation of the variability of the growth rate. J. gen. Physiol. 15, 257.

Rogers, H. J. (1953). Variant populations within a hyaluronidase-producing culture of Staphylococcus aureus. J. Path. Bact. 66, 545.

TomcsiK, J. \& GracE, J. B. (1955). Bacterial cell walls as revealed by the specific cellwall reaction and by direct staining with Alcian Blue. J. gen. Microbiol. 13, 105.

Topley and Wilson's Principles of Bacteriology and Immunity (1955), 4th edition; revised by G. S. Wilson and A. A. Miles. London: Edward Arnold.

Valentine, R. C. \& Bradfield, J. R. G. (1954). The urea method for bacterial viability counts with the electron microscope and its relation to other viability counting methods. J. gen. Microbiol. 11, 349.

Watson, G. N. (1952). The Theory of Bessel Functions, 2nd. ed. Cambridge University Press.

WiLson, G. S. (1922). The proportion of viable bacteria in young cultures with especial reference to the technique employed in counting. J. Bact. 7, 405.

YuLE, G. U. (1912). On the methods of measuring the association between two attributes. J. R. statist. Soc. 75, 579.

YULE, G. U. (1925). A mathematical theory of evolution, based on the conclusions of Dr J. C. Willis, F.R.S. Phil. Trans. B, 213, 21.

\section{EXPLANATION OF PLATE}

(a) Escherichia coli. Long organism formed by action of $m$-cresol. Phase contrast.

(b) E. coli. Same field as (a). Organisms plasmolysed by treatment with $5 \%$ NaCl. Phase contrast.

(c) Structure of Cellophane. The focal plane lies within the thickess of the membrane. Taken with a variable phase-contrast microscope adjusted for maximum contrast. 